OPEN ACCESS

Edited by:

Gabino Ríos,

Instituto Valenciano

de Investigaciones Agrarias, Spain

Reviewed by:

Heidi Halbwirth,

Vienna University of Technology,

Austria

Zhihong Zhang,

Shenyang Agricultural University,

China

*Correspondence:

Cyril Brendolise

cyril.brendolise@plantandfood.co.nz

Andrew C. Allan

andrew.allan@plantandfood.co.nz

Specialty section:

This article was submitted to

Plant Biotechnology,

a section of the journal

Frontiers in Plant Science

Received: 08 September 2017 Accepted: 12 October 2017

Published: 31 October 2017

Citation:

Brendolise C, Espley RV, Lin-Wang K, Laing W, Peng Y, McGhie T, Dejnoprat S, Tomes S,

Hellens RP and Allan AC (2017)

Multiple Copies of a Simple

MYB-Binding Site Confers

Trans-regulation by Specific Flavonoid-Related R2R3 MYBs

in Diverse Species.

Front. Plant Sci. 8:1864.

doi: 10.3389/fpls.2017.01864

\section{Multiple Copies of a Simple MYB-Binding Site Confers Trans-regulation by Specific Flavonoid-Related R2R3 MYBs in Diverse Species}

Cyril Brendolise ${ }^{*}$, Richard V. Espley ${ }^{1}$, Kui Lin-Wang', William Laing ${ }^{2}$, Yongyan Peng', Tony McGhie ${ }^{2}$, Supinya Dejnoprat ${ }^{1}$, Sumathi Tomes ${ }^{1}$, Roger P. Hellens ${ }^{3}$ and Andrew C. Allan ${ }^{1,4 *}$

${ }^{1}$ Mt Albert Research Centre, Plant and Food Research, Auckland, New Zealand, ${ }^{2}$ Fitzherbert Science Centre, Plant and Food Research, Palmerston North, New Zealand, ${ }^{3}$ Centre for Tropical Crops and Biocommodities, Queensland University of Technology, Brisbane, QLD, Australia, ${ }^{4}$ School of Biological Sciences, University of Auckland, Auckland, New Zealand

In apple, the MYB transcription factor MYB10 controls the accumulation of anthocyanins. MYB10 is able to auto-activate its expression by binding its own promoter at a specific motif, the R1 motif. In some apple accessions a natural mutation, termed R6, has more copies of this motif within the MYB10 promoter resulting in stronger autoactivation and elevated anthocyanins. Here we show that other anthocyanin-related MYBs selected from apple, pear, strawberry, petunia, kiwifruit and Arabidopsis are able to activate promoters containing the R6 motif. To examine the specificity of this motif, members of the R2R3 MYB family were screened against a promoter harboring the R6 mutation. Only MYBs from subgroups 5 and 6 activate expression by binding the R6 motif, with these MYBs sharing conserved residues in their R2R3 DNA binding domains. Insertion of the apple R6 motif into orthologous promoters of MYB10 in pear (PcMYB10) and Arabidopsis (AtMY75) elevated anthocyanin levels. Introduction of the R6 motif into the promoter region of an anthocyanin biosynthetic enzyme $\mathrm{F}^{\prime} 5^{\prime} \mathrm{H}$ of kiwifruit imparts regulation by MYB10. This results in elevated levels of delphinidin in both tobacco and kiwifruit. Finally, an $\mathrm{R} 6$ motif inserted into the promoter the vitamin $\mathrm{C}$ biosynthesis gene GDP-L-Gal phosphorylase increases vitamin C content in a MYB10-dependent manner. This motif therefore provides a tool to re-engineer novel MYB-regulated responses in plants.

Keywords: promoter, transcription factor, MYB, biotechnology, flavonoids, anthocyanin

\section{INTRODUCTION}

Gene transcription is partly controlled by transcription factors (TFs), often in combination(s) and within hierarchical networks (Hobert, 2008). These TFs interact with cis-acting sequences, which are typically found in promoters in proximity to the coding sequence of the target gene. TF proteins possess distinct functional domains, such as a DNA binding domain and a transcriptional 
activator domain. The DNA binding domain is conserved within the separate classes of TF and contain trans-acting motifs that bind to the cis-acting sequences of target gene promoters. TFs are identified by their distinct DNA binding domains, such as the large family of R2R3 domain MYB TFs (Dubos et al., 2010). Activator domains are not as conserved and are involved in the enhancement or repression of transcription, modulating the rate of mRNA synthesis by RNA polymerase II (Ptashne, 1988; Riechmann, 2002). Transcriptional regulation may depend on the interaction of binding and activator domains of different TFs, or on homo- or hetero-dimerisation of certain classes of TF proteins. TFs may co-ordinate regulation in a combinatorial manner, with different classes of TF forming complexes at the protein:DNA or protein:protein level (Grotewold et al., 2000). As TFs can control the transcription of whole pathways, they have become a target for metabolic engineering (Broun, 2004).

Identifying regulatory elements in plant promoter sequences is critical to understanding the specificity of interaction between TFs and target gene promoters, as well as understanding gene regulatory networks. The most common regulatory elements are between 6 and 15 nucleotides, which are well-conserved between species. These elements provide sites for the binding of different classes of TF proteins onto the regulatory sequences of genes to provide regulation of a particular biosynthetic pathway or transcriptional cascade. These have been characterized at the bioinformatic and functional level, and can be viewed in various databases including TRANSFAC, AGRIS, and PLACE (Wingender et al., 1996; Higo et al., 1999; Davuluri et al., 2003). Well-studied examples include the bZIP protein binding G-box (CACGTG) (Menkens et al., 1995), the MYB motifs (Sablowski et al., 1994; Romero et al., 1998), the CArG box (Riechmann et al., 1996), and combinations of these (Hartmann et al., 2005). More recently, computational approaches (Rombauts et al., 2003) and large sequencing data sets, such as the Arabidopsis 1,001-genomes project (Cao et al., 2011), have been used to expand the knowledge on regulatory elements as well as identifying new motifs (Korkuc et al., 2014).

Occasionally the targeted binding motif of a TF may also be present in the regulatory promoter region of the TF itself, providing a means of auto-regulation. The Arabidopsis MADS-box TF, AGL15, has been shown to auto-regulate its own expression by binding its own promoter sequence at cis-elements known to associate with MADS-box binding (Zhu and Perry, 2005). The Arabidopsis TT8 bHLH TF exhibits autoregulatory activity, partly mediated by members of the MYB, bHLH and WD40 (MBW) complex (Baudry et al., 2006). Further research has shown that the expression of TT8 is controlled by different MBW complexes, which dictate the synthesis of specific flavonoids in different tissues ( $\mathrm{Xu}$ et al., 2013). In apple, the anthocyanin activating R2R3 MYB, MYB10, has been shown to auto-activate its own expression by binding a cis-element in its own promoter (Espley et al., 2009). The activity of other R2R3 MYBs has been shown to be under an auto-regulatory control, with the effect sometimes being repressive. Arabidopsis MYB4, whose encoded protein is able to bind its own promoter, suppresses transcription as part of a negative auto-regulatory loop (Zhao et al., 2007). A similar repressive mechanism has been shown for the rice OsMYB4 gene (Baldoni et al., 2013), petunia MYB27 (Albert et al., 2014) and Arabidopsis AtMYBL2 (Dubos et al., 2008).

The plant MYB superfamily is abundant with over 180 members in Arabidopsis (Stracke et al., 2001) and is functionally diverse, having roles in plant development, secondary metabolism, signal transduction, disease resistance, and stress response (Jin and Martin, 1999; Dare et al., 2008). MYBs are characterized by a structurally conserved N-terminal DNA binding domain consisting of single or multiple imperfect repeats (Stracke et al., 2001). These repeats each code three alpha-helices, the third helix binding directly with the major groove of the DNA target (Stracke et al., 2001). The R2R3 MYB TFs in Arabidopsis have been divided on the basis of their sequence into 24 sub-groups (Stracke et al., 2001). More recent genome-wide studies have continued to elucidate this diverse class of TFs in other species. For example, one of the largest families has been identified in soybean, with 244 R2R3 MYBs (Du et al., 2012), while the Populus genome contains 192 R2R3 MYBs (Wilkins et al., 2009). R2R3 MYBs, acting as part of a MBW complex, are involved in flavonoid regulation including anthocyanin biosynthesis (Ramsay and Glover, 2005).

In apple, studies have shown that MYB1 (also called MYBA) is responsible for anthocyanin accumulation in the apple skin, while MYB10 regulates flesh color in some cultivars (Takos et al., 2006; Ban et al., 2007; Espley et al., 2007). Previous studies and new analysis of the recently released double-haploid 'Golden Delicious' genome confirms the allelic nature of MYB10 and MYB1/A (Lin-Wang et al., 2010; Daccord et al., 2017). In red-fleshed/red-foliaged apple accessions, the accumulation of anthocyanins correlates with an elevation of MYB10 transcript levels (Espley et al., 2007). In these cultivars there are two alleles; the MYB1 allele, which is also present in all whitefleshed, green-foliaged apples and has one copy of a 23-base pair sequence (R1) in the proximal promoter sequence of the coding region of the MYB, and secondly the MYB10 allele, which is only found in red-fleshed, red-foliage apple accessions and contains a further five copies of the 23-base pair sequence in a minisatellite-like structure (Espley et al., 2009). This multiple repeat rearrangement (R6) generates a positive auto-regulatory allele, as MYB10 can bind the 23 bp sequence as shown by in vitro binding assays (Espley et al., 2009). This is sufficient to account for the increase in MYB10 transcripts in all redfoliaged apple varieties. Further studies have confirmed that this R6-MYB10 allele is strongly associated with red-fleshed apples (Volz et al., 2009; van Nocker et al., 2011) and crab apples (Tian et al., 2015). The R6 rearrangement appears to be specific to apple as to date it has not been found naturally in red tissues of other species (e.g., plum, peach). The presence of the progenitor R1 motif is less confined, as it appears in other species in the Rosaceae family, such as pear, peach, cherry, and plum. The R6 motif can function in other species: transformation of petunia using a construct containing the apple MYB10 promoter $\left(M Y B 10-R 6_{\text {pro }}\right)$ driving the apple MYB10 gene generates increased anthocyanin in petals (Boase et al., 2015). 
Here, we demonstrate that anthocyanin-activating R2R3 MYBs from a number of species can activate the apple promoter MYB10-R6 $6_{\text {pro }}$. We show that the introduction of an R6 motif into promoters of MYB10 orthologs can initiate auto-regulation in species as diverse as pear and Arabidopsis. Engineering of this cis-element can change anthocyanin composition, harnessing the activity of different biosynthetic genes to produce a different metabolic profile. We then engineered the promoter of an enzyme completely unrelated to anthocyanin, the biosynthesis of ascorbic acid (Laing et al., 2015), to generate a gene that is then responsive to the MYB10 protein. Therefore, the R6 motif can be exploited to drive transcription of a range of genes in the presence of MYB10. This range is not restricted to flavonoid biosynthesis. This versatility could suit a range of biotechnological applications for promoter engineering in order to produce a temporal or spatial elevation of target pathways.

\section{RESULTS}

\section{MYB10 Orthologs Activate the MYB 10-R6 pro $_{\text {Promoter }}$}

We have previously reported that apple MYB10 can regulate its own transcription by binding to a characterized motif (R1) in its own promoter (Espley et al., 2009). A naturally occurring mutation of this motif, which has multiple repeats of the MYB binding site (R6), results in enhanced MYB10 auto-regulation and ectopic accumulation of anthocyanin in apple trees. Examination of many sources of apple germplasm (Espley et al., 2009) identified only R1 and R6 variants, suggesting that this mutation has happened only once. To test whether this activation is restricted to the apple MYB10, we over-expressed the anthocyanin-activating MYBs (belonging to subgroup 6, according to Stracke et al., 2001) from pear, strawberry, Arabidopsis, petunia, and kiwifruit in Nicotiana benthamiana together with the R1 or R6 versions of the apple MYB10 promoter fused to a luciferase reporter (Hellens et al., 2005). Expression of these MYBs and their respective bHLH partner led to an average 16-fold activation of the R6 variant of the MYB10 promoter compared with R1 (Figure 1). Some of the MYBs tested, particularly PhDPL, were able to activate the R6 promoter to a greater extent than MYB10 from apple. In the same experiment, MdMYB8, an R2R3 MYB from apple, which is not implicated in anthocyanin regulation (Espley et al., 2007), failed to activate the R6 motif, even in the presence of a bHLH partner.

\section{Flavonoid-Related R2R3 MYBs Activate the $M Y B 10-R 6_{\text {pro }}$ Promoter}

Anthocyanin-related MYBs from a diverse range of species activated the R6 promoter, whereas MdMYB8, not implicated in anthocyanin regulation, failed to do so. To further test this specificity, the R1 and R6 variants of the MdMYB10 promoter were screened with a panel of apple R2R3 MYBs. To ensure that we tested MYBs across a range of subgroups (Stracke et al., 2001), all the available apple MYB sequences were extracted from the apple genome database (Velasco et al., 2010). These were then assembled into a phylogenetic tree together with Arabidopsis MYBs. From this, a total of 29 R2R3 MYBs from a diverse range of subgroups were selected.

Among all the apple MYBs tested, only MYB9, MYB10, MYB11, MYB12, and MYB110 were able to activate the $M Y B 10-R 6_{\text {pro }}$ promoter (Figure 2A) in the presence of MdbHLH3, suggesting that recognition of the R6 motif was restricted to flavonoid-related MYBs (Figure 2B). These MYBs are from subgroup 5 (MdMYB9 and 11), representing the proanthocyanidin MYB clade (e.g., Arabidopsis TT2), subgroup 6 (MdMYB10 and 110) representing the anthocyanin-associated clade (e.g., AtMYB75) and a putative ortholog of AtMYB5 (MdMYB12), which regulates seed mucilage and seed-coat pigmentation (Li et al., 2009).

\section{Conserved Amino Acid Residues within the R6-Activating MYBs}

Protein alignment of the R2R3 domain of all the MYBs tested in the transient assay (Figures 1, 2), revealed that the flavonoid related MYBs shared a potential amino acid signature in their R2R3 domain consisting of a Lysine or Arginine residue at position 25 and a Lysine, Arginine, or Glutamine residue at position 72, relative to MdMYB10 sequence (Figure 3 ). We also included in our analysis the sequences of other MYBs not necessarily related to flavonoid biosynthesis (Figure 3). In these, the signature $(\mathrm{K} / \mathrm{R})_{25}-(\mathrm{X}) \times 46-(\mathrm{K} / \mathrm{R} / \mathrm{N})_{72}$ was not apparent at these positions, supporting a specificity linked to flavonoid related MYBs.

\section{Insertion of the R6 Motif into the Promoters of Pear and Arabidopsis}

The specificity of the activation of the R6 motif to MYB10 orthologs and other flavonoid-related MYBs suggests that this motif could be used to elevate plant anthocyanin levels in species other than apple. To test this, the motif was inserted into the promoter of MYB10 orthologs from a close apple relative, European pear (PcMYB10) and an unrelated species, Arabidopsis (AtMYB75). The promoter of PcMYB10 naturally harbors a single repeat of the $\mathrm{R} 1$ motif at a similar distance to the start codon as that seen in the apple MYB10 promoter. The R6 motif was inserted upstream of the predicted 5 ' UTR region in the promoter of pear and Arabidopsis, fused to the luciferase reporter (schematic in Figure 4A). Transient assays in $N$. benthamiana revealed that both pear and Arabidopsis $M Y B$ promoters harboring the R6 motif were strongly activated by MdMYB10, PcMYB10, or AtMYB75 (Figure 4B). This activation was dependent on a bHLH, either from apple (MdbHLH3) or Arabidopsis (AtbHLH2/EGL3). For the engineered promoters of either pear or Arabidopsis, the level of activation was similar when co-infiltrated with MdMYB10 or with their own MYB activator, PcMYB10 or AtMYB75, respectively.

The engineered promoters harboring the R6 motif were then used to drive the expression of their own respective genes (PcMYB10 and AtMYB75), rather than a luciferase 

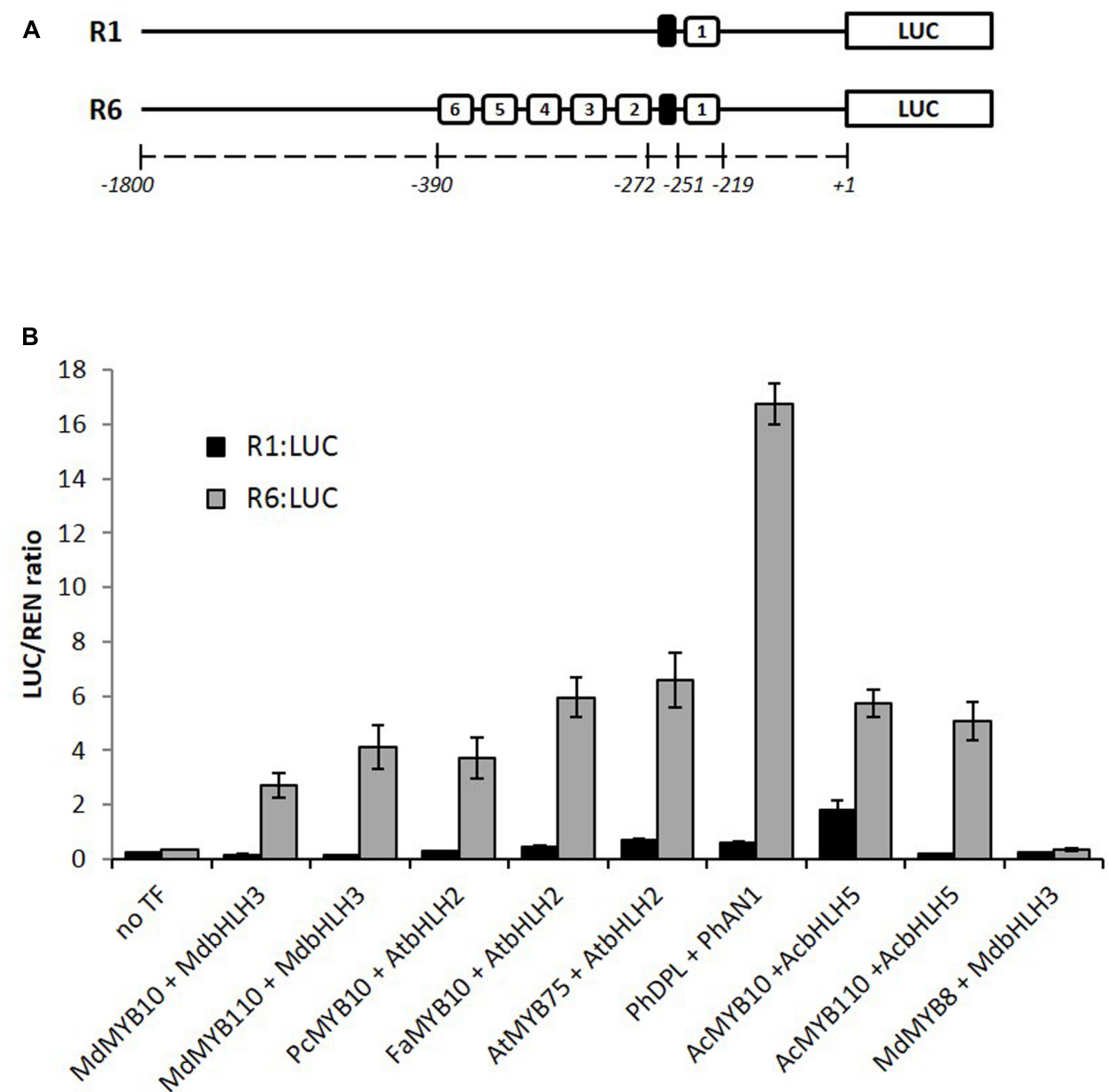

FIGURE 1 | MYB10 orthologs activate the R6-containing MYB10 promoter in a transient assay in Nicotiana benthamiana. (A) Schematic of the R1 and R6 apple MYB10 promoters fused to the LUC reporter gene in pGreen0800LUC representing the R repeat units (open boxes) and the microsatellite structure upstream of unit 1 (black box) and their position relative to the ATG start codon. (B) Leaves of N. benthamiana were infiltrated with the MdMYB10-R1 pro:LUC or

MdMYB10-R6 pro:LUC fusions (named R1:LUC and R6:LUC respectively) on their own (no TF) or co-infiltrated with MdMYB10 or MYB10 orthologs and bHLH partner. Md: Malus domestica, Pc: Pyrus communis, Fa: Fragaria ananassa, At: Arabidopsis thaliana, Ph: Petunia hybrida, Ac: Actinidia chinensis. Luminescence was measured 3 days post-infiltration and is expressed as a ratio of the LUC to REN signals. Data represent means ( \pm SE) of four technical replicate reactions.

reporter. Three constructs were transformed in Arabidopsis: the native MYB75 promoter driving MYB75 genomic DNA (termed MYB75 pro:MYB75), the engineered MYB75-R6 promoter driving MYB75 (termed MYB75-R6 pro:MYB75), and a $35 S_{\text {pro }}: M Y B 75$ construct (Figure 5A) previously shown to elevate the anthocyanin content in Arabidopsis (Borevitz et al., 2000). Three independent $\mathrm{T} 2$ generation lines were generated for each construct. The MYB75-R6 pro:MYB75 lines showed enhanced pigmentation compared with MYB75 pro:MYB75, increasing from $1.3 \mu \mathrm{g} / \mathrm{g} \mathrm{FW}$ to $26.6 \mu \mathrm{g} / \mathrm{g} \mathrm{FW}$ (Figure 5B). However, this is not as pigmented as the $35 S_{\text {pro: }}$ MYB75 lines (Figure 5B; $147.8 \mu \mathrm{g} / \mathrm{g}$ FW). Expression analysis of these lines revealed an eightfold increase in $M Y B 75$ expression in the MYB75-R6 $6_{\text {pro }}: M Y B 75$ lines compared with MYB75 pro:MYB75 (Figure 5C). Analysis of anthocyanins in these lines revealed that the total anthocyanin content measured in each line was related to the level of MYB75 transcription (Figure 5D).
The pear MYB10 construct engineered to contain the R6 motif was transformed into 'Conference' pear. The multiple lines generated showed varying levels of foliar anthocyanin content both visually and using HPLC analysis, whereas no anthocyanin was detected in mature leaves of wild-type pear grown under the same glasshouse conditions (Figures 6A,B). The expression of PcMYB10 was elevated in the presence of the R6 motif compared with the wild-type line (Figure 6C). These results showed that the insertion of the R6 motif in the promoter of AtMYB75 or PcMYB10 is able to generate an auto-regulatory allele, leading to an ectopic accumulation of anthocyanins in the plant.

\section{Manipulating Anthocyanin Profile Using the R6 Motif}

We examined the potential of using the R6 motif to modify a known biosynthetic pathway by modifying the expression of 


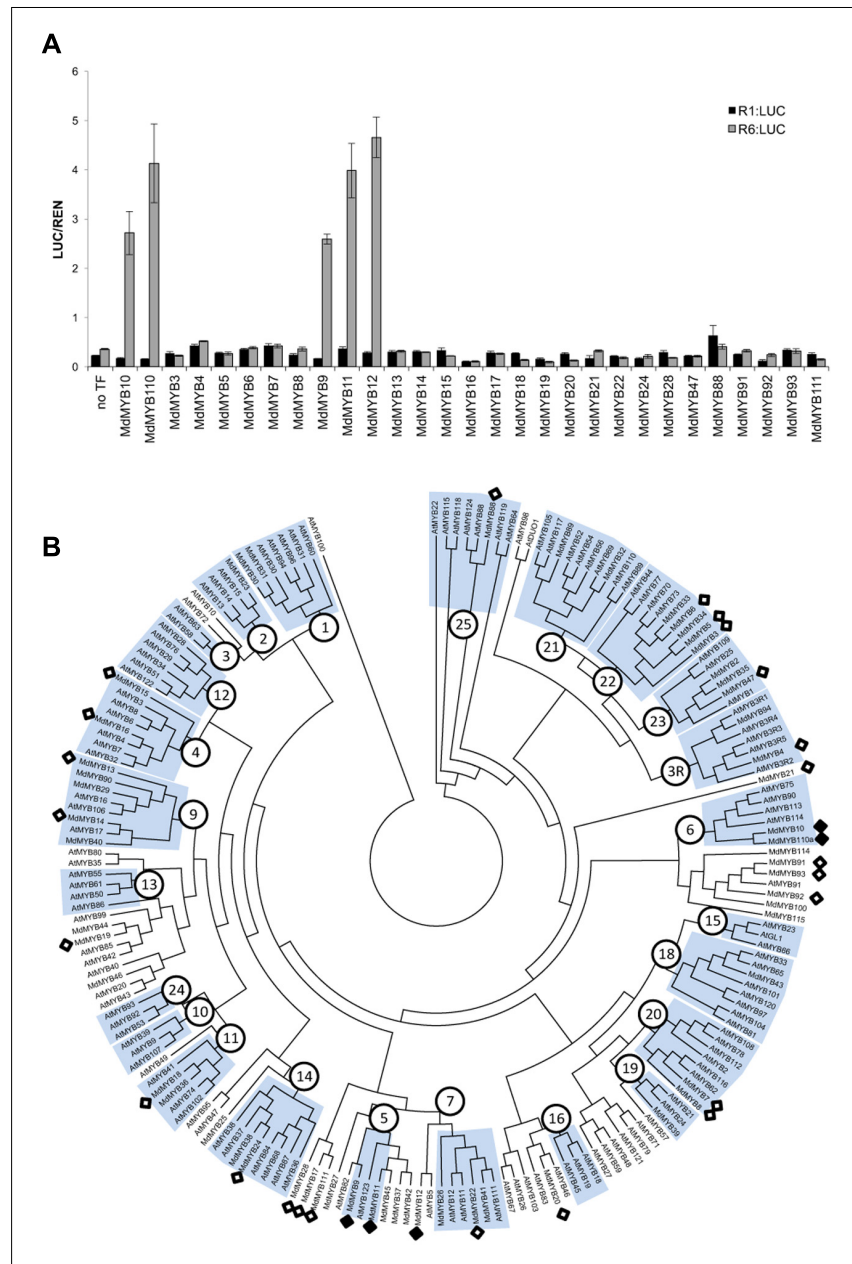

FIGURE 2 | Apple MYBs from subgroups 5, 6 and MdMYB12 can activate the R6-containing MYB10 promoter. (A) Apple MYB transcription factors (TFs) were co-infiltrated with MdbHLH3 and the MdMYB10-R1 pro:LUC or MdMYB10-R6 pro:LUC fusions (named R1:LUC and R6:LUC respectively) in $N$. benthamiana leaves and the LUC/REN signal was measured. Data represent means ( \pm SE) of four technical replicate reactions. (B) Phylogenetic tree representing relationships between apple and Arabidopsis MYBs. Numbers indicate subgroups previously described for Arabidopsis MYBs (Stracke et al., 2001). Apple MYBs tested in transient assay in (A) (white diamond) and the MYBs tested positive for transactivation of the R6 promoter (black diamond) are indicated. Deduced amino acid sequences were aligned using CLUSTALX. Protein distances were calculated with PROTDIST using the Jones-Taylor-Thornton matrix and the tree was constructed by the neighbor-joining method.

key genes. The hue of anthocyanin pigments depends on their level of hydroxylation and the position of the hydroxyl group on the B-ring (Harborne, 1967). These hydroxylation steps are directed by $\mathrm{P} 450$-dependent monooxygenases, the flavonoid $3^{\prime}$-hydroxylase $\left(\mathrm{F}^{\prime} \mathrm{H}\right)$ and the flavonoid $3^{\prime} 5^{\prime}$-hydroxylase $\left(\mathrm{F}^{\prime} 5^{\prime} \mathrm{H}\right)$, responsible for the synthesis of cyanidin and delphinidin derivatives respectively. In kiwifruit (Actinidia sp.), delphinidin derivatives have been identified in two pigmented species, $A$. melanandra and A. arguta var. purpurea (Montefiori et al., 2009), but not in commercially grown green (A. deliciosa) and yellow or red (Actinidia chinensis) fruit.
The promoter region of the $F 3^{\prime} 5^{\prime} H$ gene was isolated from genomic DNA of the yellow kiwifruit and fused to the luciferase reporter gene, with or without a R6 motif inserted 183 bp upstream of the start codon (Supplementary Figure S1A). Using a transient assay in N. benthamiana, apple MdMYB10 and kiwifruit AcMYB110 (Fraser et al., 2013) were able to enhance the activation of the engineered promoter harboring the R6 motif by threefold compared with the native $F 3^{\prime} 5^{\prime} H$ promoter (Supplementary Figure S1B). The engineered R6 promoter was then fused to the genomic DNA of the $F 3^{\prime} 5^{\prime} \mathrm{H}$ gene to generate the $F 3^{\prime} 5^{\prime} H-R 6_{\text {pro }}: F 3^{\prime} 5^{\prime} H$ construct, which was transiently expressed together with MYB110 in tobacco leaves (N. tabacum). Anthocyanin measurements revealed that MYB110 induced a 6.7-fold increase of the $\mathrm{Dp} / \mathrm{Cy}$ (derivatives) ratio when the R6 motif was inserted in the $\mathrm{F}^{\prime} 5^{\prime} \mathrm{H}$ promoter compared with the native promoter (Supplementary Figures S1C,D). MYB110 alone induces synthesis of approximately equal amounts of cyanidin (Cy-glucoside, Cy-rutinoside) and delphinidin derivatives (Dp-rutinoside) in tobacco leaves. The higher Dp/Cy ratio obtained with MYB110 plus $F 3^{\prime} 5^{\prime} H-R 6_{p r o}: F 3^{\prime} 5^{\prime} H$ construct was due to a reduced amount of cyanidin derivatives, while content of delphinidin derivatives were similar. This could suggest that the enhanced $\mathrm{F}^{\prime} 5^{\prime} \mathrm{H}$ activity competed with the endogenous $\mathrm{F}^{\prime} \mathrm{H}$ of tobacco, hence reducing the amount of substrate available for cyanidins.

We also assessed whether the number of repeats had an effect on the magnitude of promoter activation. The kiwifruit $F 3^{\prime} 5^{\prime} H$ promoter was engineered with additional copies of the R6 motif, generating promoters harboring 6, 12, or 30 units of the R1 sequence (Figure 7A). These promoters, fused to the luciferase reporter or to the $F 3^{\prime} 5^{\prime} H$ genomic coding sequence, were tested in $N$. benthamiana and tobacco. The luciferase reporter assay suggested that maximum activation is obtained with 12 to 30 repeats, while the Dp/Cy ratio measured in tobacco leaf showing 12 repeats gave the highest ratio (Supplementary Figure S2).

To validate this result in kiwifruit tissue, the same constructs were assayed by injection of Agrobacterium carrying cloned genes into $A$. eriantha fruits (Figure 7B) (Montefiori et al., 2011). Fruits expressing MYB110 alone produced cyanidinbased compounds and trace amounts of delphinidin-galactoside and delphinidin-xylogalactoside (Figure 7C). Co-infiltration of the $\mathrm{F3}^{\prime} 5^{\prime} \mathrm{H}_{\text {pro }}: \mathrm{F3}^{\prime} 5^{\prime} \mathrm{H}$ construct slightly enhanced delphinidin derivative levels. However, a significant increase of delphinidins was measured when the engineered $F 3^{\prime} 5^{\prime} H-R 6_{\text {pro }}: F 3^{\prime} 5^{\prime} H$ was coinfiltrated, yielding to a $\mathrm{Dp} / \mathrm{Cy}$ ratio 8 times higher than with the native promoter (Figure 7D).

\section{Elevating Ascorbate Content in Plants Using the R6 Motif}

Ascorbate (or vitamin C) is an essential metabolite for most living organisms. The GDP-L-galactose phosphorylase, encoded by the GGP gene (also known as VTC2) has been shown to be the main control point of the pathway (Bulley et al., 2009; Laing et al., 2015). This gene is regulated transcriptionally during fruit development, but also post-transcriptionally via a highly conserved UORF within its $5^{\prime}$ UTR region that represses its 


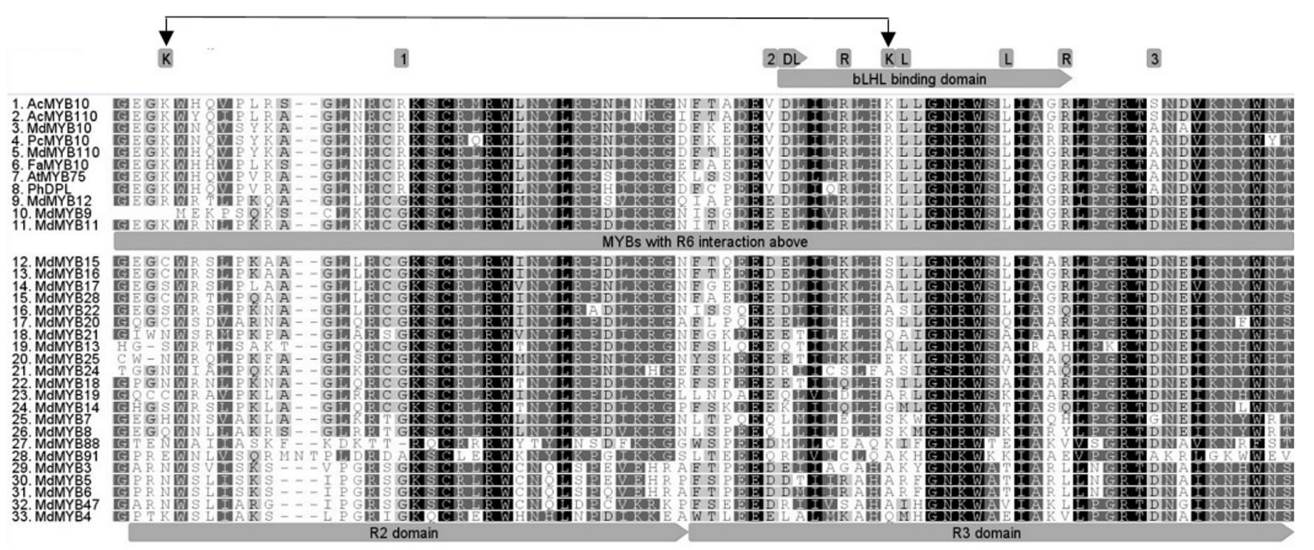

FIGURE 3 | Deduced amino acid sequences were aligned using CLUSTALW. The bHLH binding region within the R3 domain is indicated (gray arrow) and conserved amino acids required for the bHLH-dependant activation (Grotewold et al., 2000) are highlighted above it. Box 1, 2, and 3 show previously described conserved residues specific to dicot anthocyanin-promoting MYBs (Lin-Wang et al., 2010). Double black arrow indicates the signature $(\mathrm{K} / \mathrm{R})_{25}-(\mathrm{X}) \times 46-(\mathrm{K} / \mathrm{R} / \mathrm{N})_{72}$ specific to the R6-activating MYBs.

A

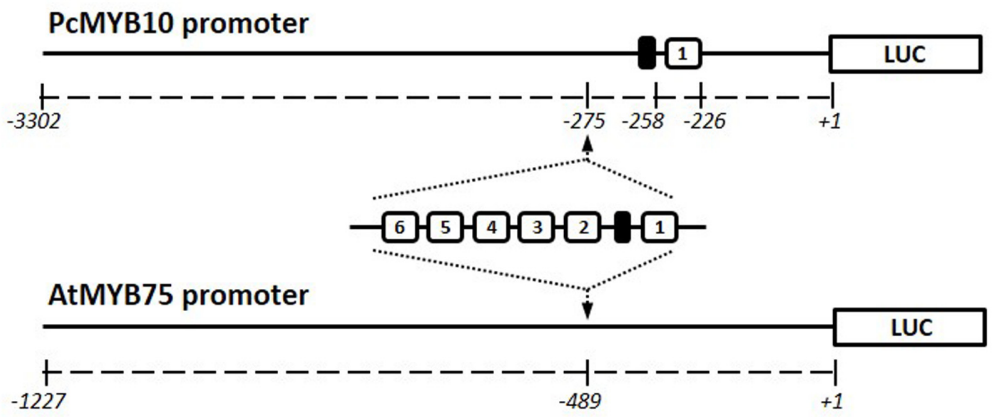

B
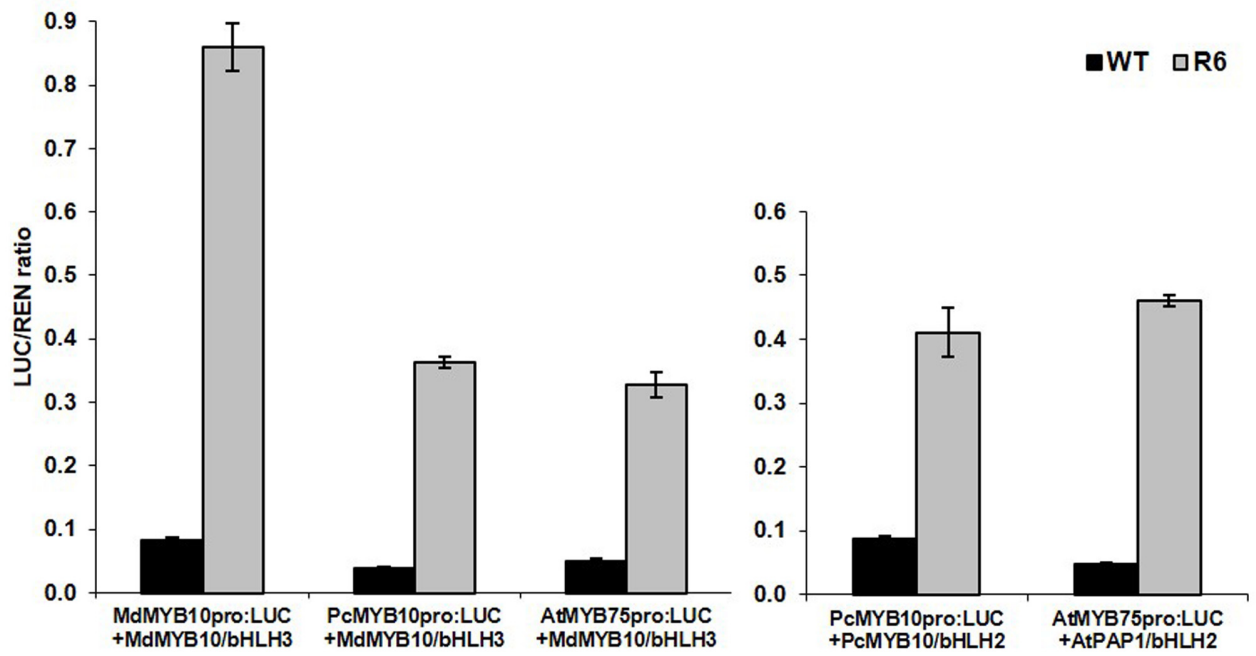

FIGURE 4 | Insertion of the R6 domain in the promoter of MYB10 orthologs leads to autoregulation. (A) Schematic of the engineered pear MYB10 and AtMYB75 promoters fused to the LUC reporter gene in pGreen0800LUC. (B) Dual luciferase promoter assay of the R6 engineered promoters in $N$. benthamiana. Promoters, with or without the R6 domain, were co-infiltrated with MdMYB10 and MdbHLH3, PcMYB10 and AtbHLH2, or AtMYB75 and AtbHLH2. Luminescence of LUC and REN was measured 3 days post-infiltration and expressed as a ratio of LUC to REN. Data represent means ( \pm SE) of four technical replicate reactions. 


\section{A}

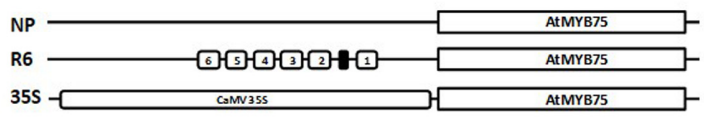

B

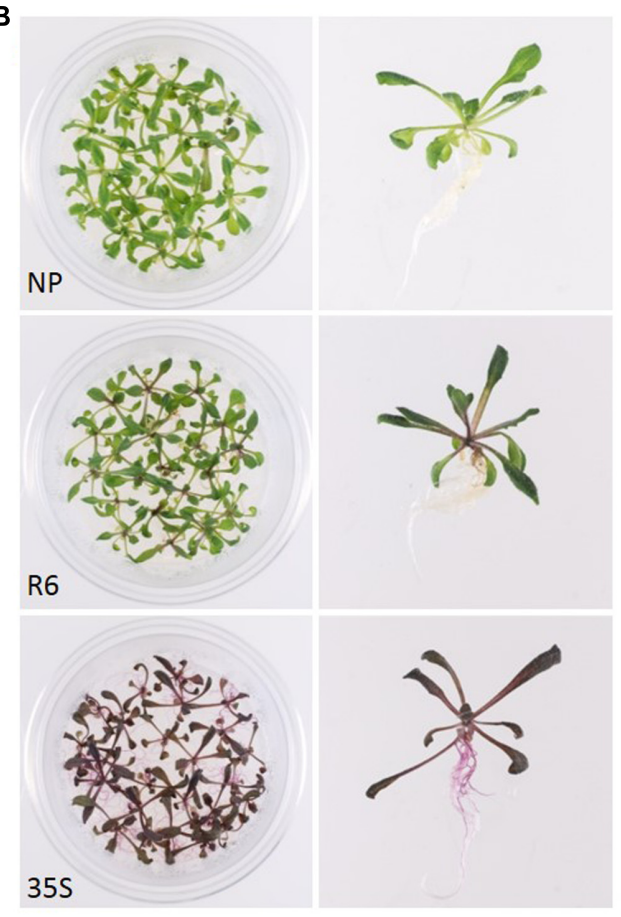

C

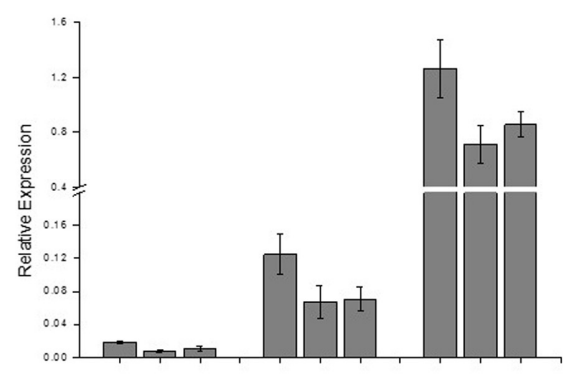

D

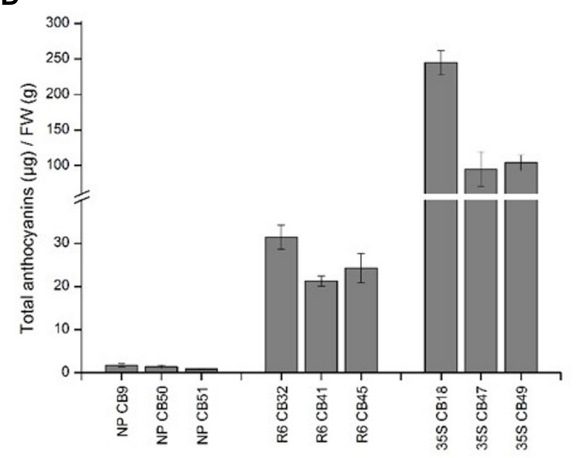

FIGURE 5 | Insertion of the R6 motif into the promoter of AtMYB75 induces AtMYB75 overexpression and ectopic anthocyanin accumulation. (A) Schematics of the engineered AtMYB75 constructs transformed into Arabidopsis. (B) Phenotype of representative T2 lines transformed by AtMYB75 pro:AtMYB75 [denoted as native promoter (NP)], AtMYB75-R6 pro:AtMYB75 (denoted as R6) and 35S pro:AtMYB75 (denoted as 35S). (C) Expression analysis by qPCR of the transcripts of the AtMYB75 gene in each transformed lines. Expression is given relative to actin and error bars represent the standard errors of the means calculated from four technical replicates. (D) Total anthocyanin content in each lines measured by HPLC and expressed as $\mu \mathrm{g}$ of Cy-glu equivalent per gram of fresh weight (FW).

translation under high ascorbate concentration (Laing et al., 2015).

The GGP promoter sequence was isolated from kiwifruit (A. eriantha), fused to the LUC reporter gene and the R6 motif was inserted upstream of the $5^{\prime}$ UTR (Figure 8A). Transient expression in $N$. benthamiana revealed that MdMYB10 was able to activate the GGP promoter harboring the R6 motif (Figure 8B). To test for any potential increase in ascorbate levels, further assays were performed in $N$. benthamiana using the CaMV35S promoter driving GGP and a version of the GGP promoter engineered with two copies of the R6 motif fused to the GGP cDNA (named GGPwt-R12 pro:GGP). These were co-infiltrated in the presence of either the kiwifruit MYB110 or the apple MYB8 as a control. Ascorbate content of the infiltrated patch revealed that the GGP construct harboring the R12 motif (GGPwt-R12 pro:GGP) did not increase the ascorbate content (Figure 8C). This is likely due negative feedback repression by ascorbate involving the $\mathrm{UORF}$ in the $5^{\prime}$ UTR of the GGP promoter (Laing et al., 2015). It was shown recently that a single point mutation of the non-canonical start codon of the uORF was sufficient to cancel this negative feedback (Laing et al., 2015).
Therefore, we made the same point mutation in the two versions of the GGP promoter, either with or without the R12 motif. The construct harboring the R12 motif and the mutated UORF (GGPmut-R12 pro:GGP) was able to induce over-expression of GGP in the presence of MYB110, resulting in a fivefold increase in ascorbate content in tobacco leaves (Figure 8C). In the presence of control MdMYB8, no increase in ascorbate concentration was observed. The 35S-driven construct was able to elevate ascorbate levels, irrespective of MYB co-infiltration.

\section{DISCUSSION}

The transcriptional regulation of endogenous genes and pathways by TFs is one key approach for plant biotechnology. The promoters of targeted genes must respond to the TF, which is dependent on the presence of cis-regulatory elements. Synthetic promoter engineering is designed to produce promoters that offer stronger and more precisely targeted transcription (Venter, 2007). Engineering is still reliant on known cis-elements, hence the discovery of new enhancer or repressor elements 
A
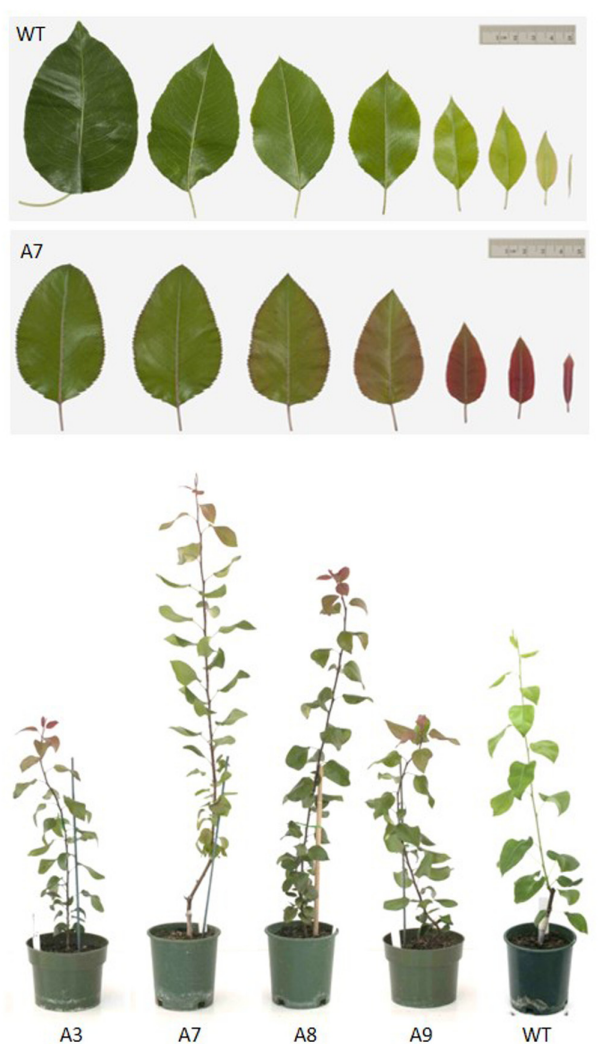
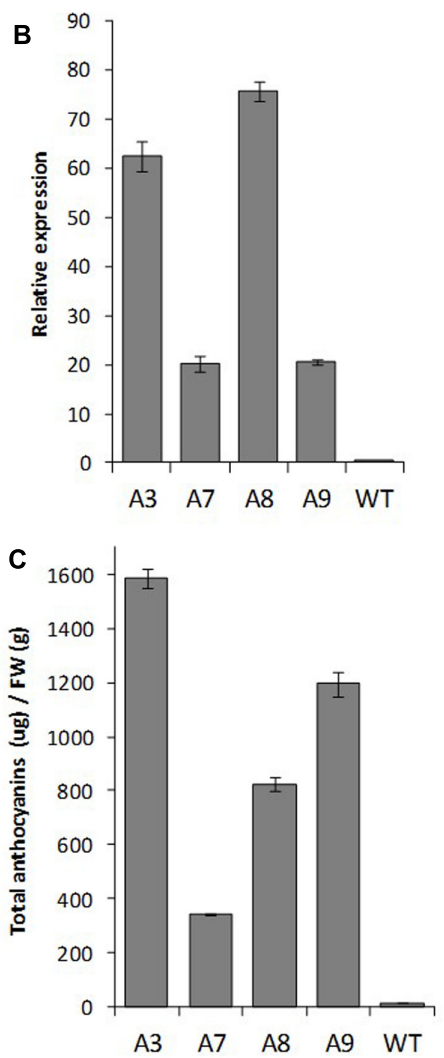

FIGURE 6 | Insertion of R6 domain into the pear MYB10 promoter induces PcMYB10 overexpression and ectopic anthocyanin accumulation. (A) Phenotype of representative transgenic pear lines harboring the PCMYB10-R6 pro:PCMYB10 construct. (B) Expression analysis by qPCR of the transcripts of the PCMYB10 gene in fully expanded mature leaves of each transgenic line. Expression is given relative to pear actin and error bars represent the standard errors of the means calculated from four technical replicates. (C) Total anthocyanin content of fully expanded mature leaves of each line measured by HPLC and expressed as $\mu \mathrm{g}$ of Cy-glu equivalent per gram of fresh weight (FW). Error bars represent the standard errors of the means calculated from three replicates.

is important. A successful product will also depend on the social and regulatory issues surrounding emerging new gene synthesis and editing technologies, and the outcome of the trans-genesis versus cis-genesis debate (Holme et al., 2013; Doudna and Charpentier, 2014). Here we describe a number of applications for the R6 motif from apple, a naturally occurring mutation, which produces enhanced binding for specific MYB TFs. This can be used to upregulate biosynthetic pathways or manipulate metabolite composition, with potential for either cis-genic adoption in apple and pear, or gene editing technologies in other -species. We describe diverse applications, to increase anthocyanin levels (e.g., pear which often suffers from reduced color), alter anthocyanin profile (e.g., kiwifruit, a relatively un-domesticated crop to add novelty and potential health targets), or enhance other metabolites (e.g., vitamin C, a major health target).

Apple MYB10 is a member of a distinct clade of dicot R2R3 MYBs that activate the expression of anthocyanin biosynthetic genes. MYB10 is able to auto-activate its expression by binding its own promoter at an R1 motif, with a natural mutation of this motif, termed R6, resulting in stronger auto-activation. MYB10 orthologs from Arabidopsis, petunia and kiwifruit, representing the Brassicaceae, Solanaceae and Actinidiaceae families, also activate R6. Petunia MYB DPL, in particular, was able to transactivate the apple promoter to a high level. Corresponding cis-elements in these species, which resemble R1 or R6-like motifs, were seen in promoters of apple, pear and Prunus species, but less evident outside the Rosaceous family (Supplementary Figure S3). Activation required the co-infiltration of a bHLH partner.

In a number of plant species, ectopic expression of anthocyanin accumulation has been observed and is associated with altered expression of an anthocyanin-activating MYB. In cauliflower, a Harbinger DNA transposon insertion into the promoter of BoMYB2 causes ectopic anthocyanin accumulation in the curd (Chiu et al., 2010). In red-foliaged plum (Prunus cerasifera) PcMYB10.6 is constitutively up-regulated and is responsible for this phenotype ( $\mathrm{Gu}$ et al., 2015). A similar, but independent event is responsible for red foliage in peach ( $P$. persica) where PpMYB10.4 (a paralogous gene) is mis-regulated (Zhou et al., 2014). However, none of these phenotypes is the result of an R6-like mutation. Despite this, the apple motif is recognized by MYBs from other species. 


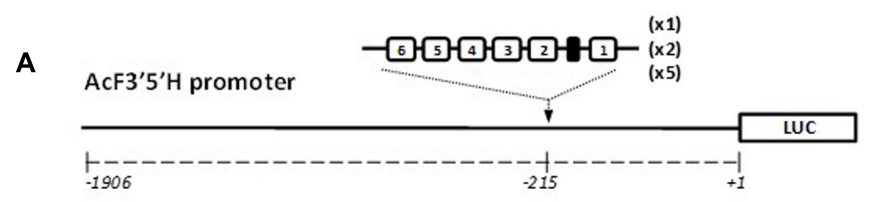

B
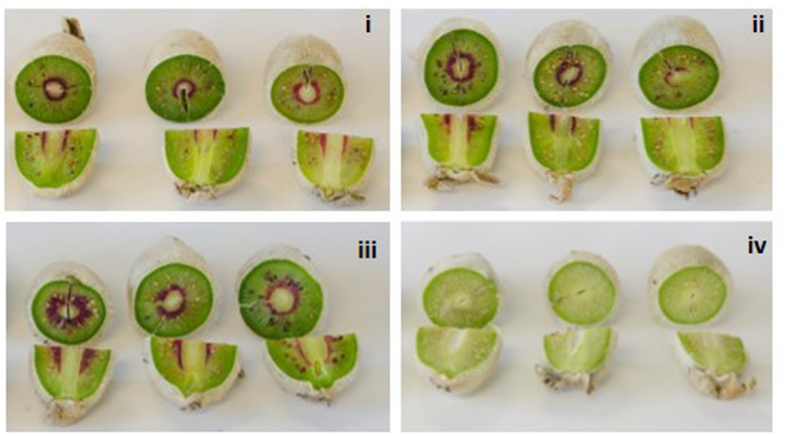

C

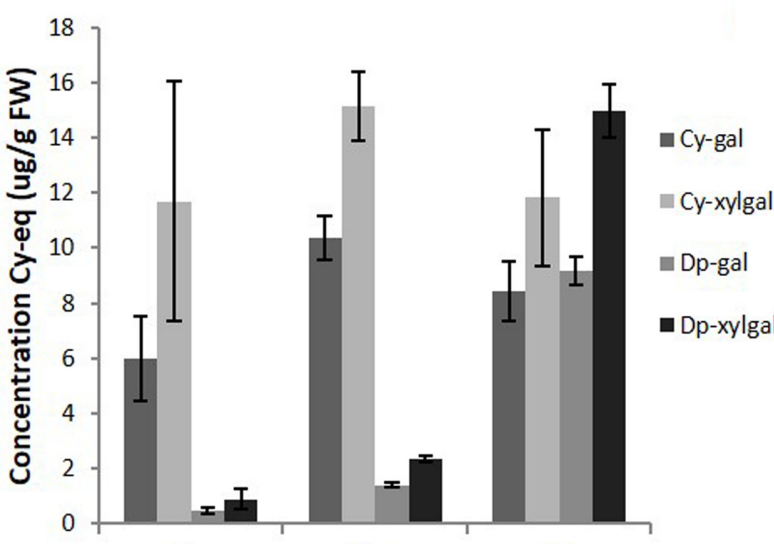

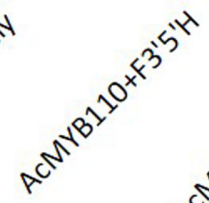

D

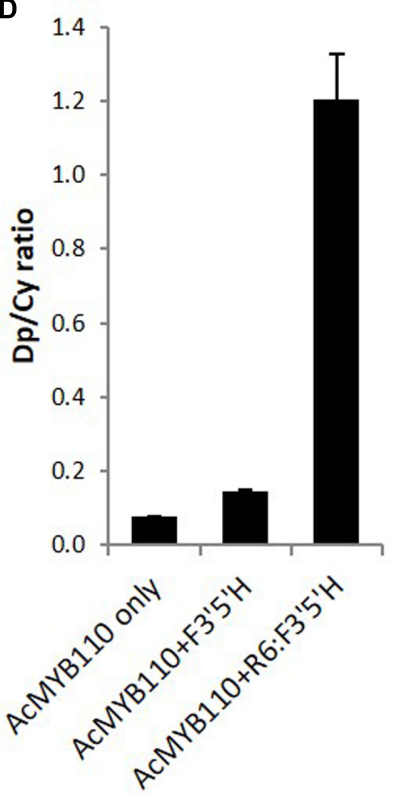

FIGURE 7 | Insertion of the R6 motif into the $A c F 3^{\prime} 5^{\prime} H$ promoter induces accumulation of delphinidin derivatives. (A) Schematic of the engineered $\mathrm{F} 3^{\prime} 5^{\prime} \mathrm{H}$ promoter fused to the LUC reporter gene in pGreen0800LUC. (B) Actinidia eriantha kiwifruit were infiltrated by Agrobacterium suspension containing AcMYB110 (i),

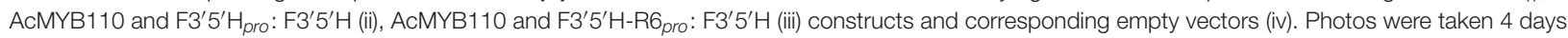
post-infiltration. (C) Measurement of cyanidin and delphinidin derivatives in kiwifruits expressing corresponding constructs, expressed as concentration of cyanidin-gal equivalent and (D) presented as a ratio of delphinidin to cyanidin.

Previous studies show the specificity of certain subgroups of R2R3 MYBs for regulation of specific flavonoids, such as in Arabidopsis (Stracke et al., 2001), peach (Ravaglia et al., 2013) and reviewed in Lai et al. (2013). These include a number of conserved features including the bHLH binding domain (Grotewold et al., 2000) and residues that specify the regulation of either anthocyanins or proanthocyanidins (Heppel et al., 2013). This latter study provided an understanding into the evolutionary aspects of different regulators from a common ancestral MYB from functional analysis of residues within the R2R3 domain. Of the 29 apple MYBs tested across a range of phylogenetic clades, only those belonging to known flavonoid-related subgroups were able to activate the R6 motif. No activation was observed for the previously reported apple anthocyanin repressors, such as MdMYB111 or MdMYB17 (SG4) (Lin-Wang et al., 2010) or for the flavonol regulator MdMYB22 (SG7) (Peng et al., 2013). A consensus signature of $(\mathrm{K} / \mathrm{R})_{25}-\mathrm{Nx} 46-(\mathrm{K} / \mathrm{R} / \mathrm{N})_{72}$ is present in MYBs that trans-activated the R6 motif. This suggests evolution of key amino acids specifying DNA motif recognition. The mechanism for this should be examined in further studies.

We tested R6 in stable transformations of both pear and Arabidopsis to confirm their effect on anthocyanin biosynthesis. 
A 65-4-3
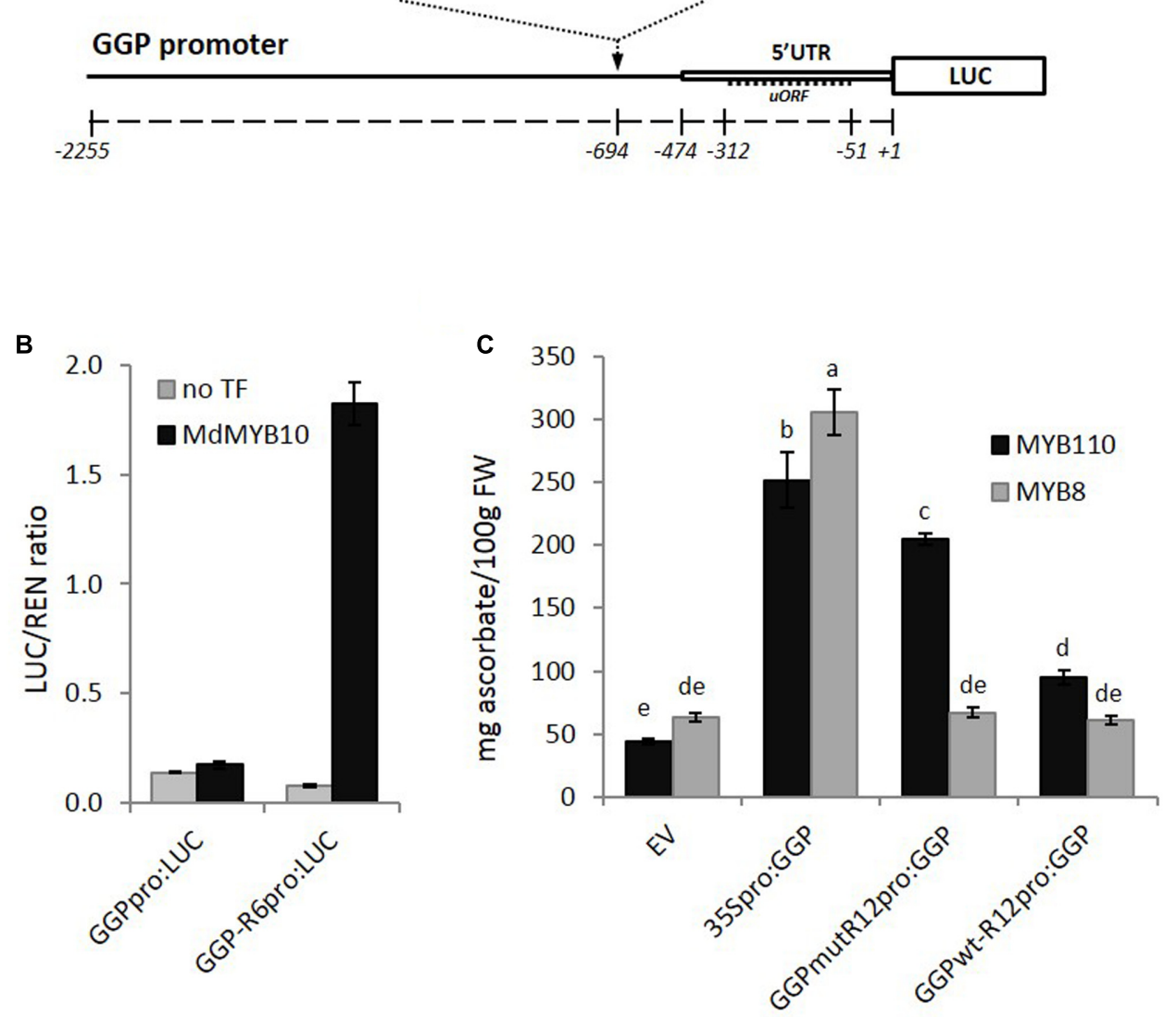

FIGURE 8 | Effect of inserting the R6 motif in the GGP promoter. (A) Schematic of the engineered GGP promoter fused to the LUC reporter gene in pGreen0800LUC representing the position of two R6 insertions upstream of the $5^{\prime}$ UTR and uORF (open box and dash line respectively). (B) Dual luciferase assay of the GGP promoter harboring the R6 motif fused to the LUC reporter. Promoter constructs were co-infiltrated in N. benthamiana with MdMYB10 and MdbHLH3. Luminescence of LUC and REN was measured 3 days post-infiltration and expressed as a ratio of LUC to REN. Data represent means ( \pm SE) of four replicate reactions. (C) The GGP cDNA, placed under its own promoter engineered with two copies of the R6 motif and harboring or not a single point mutation of the uORF start codon (GGPmut-R12 pro:GGP and GGPwt-R12 pro-GGP, respectively), or placed under the control a $35 S$ promoter (35S pro:GGP), or the corresponding empty vector (EV) were co-infiltrated with AcMYB110 (or the MYB8 negative control). Ascorbate content was measured at 5 dpi. Data represent means ( \pm SE) of six biological replicates. One-way ANOVA test was applied and resulting grouping information was obtained using the Tukey method, columns sharing the same letters are not significantly different at $P<0.05$.

The Arabidopsis promoter containing R6 fused to the AtMYB75 coding sequence was transformed into Arabidopsis (Figure 5). The stable R6-containing lines showed increases in anthocyanin, which was confirmed by HPLC analysis. The transcription level of AtMYB75 was also clearly elevated in these lines. Transformation of pear with an engineered version of the PcMYB10 gene containing the R6 motif produced trees with ectopic anthocyanin accumulation in the leaves. Both PcMYB10 transcription and anthocyanin concentration were elevated, compared with undetectable levels in WT tissue. This is the first reported case of manipulation of pear pigmentation using stable transformation.

The function of MYB10 is to bind the promoters of the genes encoding enzymes of the anthocyanin biosynthetic pathway to control pigment levels. This has previously been shown to occur through MYB binding sites (Sablowski et al., 1994).
To determine if insertion of an R6 motif would enhance the binding capacity of MYB10 to biosynthetic promoters, the promoter of the $\mathrm{F}^{\prime} 5^{\prime} \mathrm{H}$ from kiwifruit was engineered. Trans-activation in tobacco occurred with both the apple and kiwifruit anthocyanin-related MYBs (when co-infiltrated with a bHLH), which was increased 12-fold after insertion of an R6 motif compared with the background activity of the promoter. Despite this R6-driven increase, the total concentration of anthocyanin in the tobacco leaf remained relatively constant. However, the anthocyanin composition was changed: infiltration with the $F 3^{\prime} 5^{\prime} H-R 6_{\text {pro }}: F 3^{\prime} 5^{\prime} H$ or $35 S_{\text {pro }}: F 3^{\prime} 5^{\prime} H$ produced a shift from cyanidin derivatives to delphinidin derivatives. This is consistent with the function of $\mathrm{F}^{\prime} 5^{\prime} \mathrm{H}$ (Seitz et al., 2007). The greatest change in ratio was achieved with the R6 engineering, suggesting that this is an efficient way of re-directing anthocyanin composition. Transient assays of $\mathrm{F}^{\prime} 5^{\prime} \mathrm{H}$ were performed in 
fruit tissue of kiwifruit (Figure 7). We compared the effect of infiltrating $A c M Y B 110$ alone or with the endogenous kiwifruit $F 3^{\prime} 5^{\prime} \mathrm{H}$ promoter driving the $F 3^{\prime} 5^{\prime} \mathrm{H}$, or with the R6-containing $F 3^{\prime} 5^{\prime} H$ promoter driving the $F 3^{\prime} 5^{\prime} H$. There was an increase in total anthocyanin concentration with $F 3^{\prime} 5^{\prime} H-R 6_{p r o}: F 3^{\prime} 5^{\prime} H$ together with a dramatic shift from cyanidin derivatives to delphinidin derivatives. Several studies have targeted the content of cyanidin and delphinidin derivatives in plants in order to modify color, which is usually achieved by either overexpressing or silencing the $\mathrm{F}^{\prime} 5^{\prime} \mathrm{H}$ enzyme, such as in carnations (Fukui et al., 2003), roses (Katsumoto et al., 2007), and tobacco (Okinaka et al., 2003). The color of fruit is also of interest to plant breeders and being able to modify the pigment content in fruit skin or flesh is a valuable tool for breeding new varieties, such as in apple (Volz et al., 2009).

To test the R6 motif in a completely unrelated biosynthetic pathway, we engineered the vitamin $\mathrm{C}$ pathway gene, GGP, to include the R6 motif. Humans lack the ability to synthesize vitamin $\mathrm{C}$ and rely on ingestion from plants. Being able to increase ascorbate content in plants has become an important target for plant breeding. Therefore, the ascorbate biosynthetic pathway and its regulation have been extensively studied (Bulley and Laing, 2016). Vitamin C levels regulate translation of the key gene, GGP, via a repression at a uORF within the $5^{\prime} \mathrm{UTR}$ of the gene (Laing et al., 2015). Insertion of an R6 into the GGP promoter was ineffectual while this uORF was intact. However, when the UORF was disrupted by a point mutation in the non-canonical start site, vitamin $\mathrm{C}$ levels were highly elevated in tobacco leaves when AcMYB110 was co-infiltrated with the R6-containing GGP promoter. This shows that the ability of the MYB protein to bind promoter sequences harboring R6 is not restricted to flavonoid-related genes and supports the hypothesis that MYB10 can enhance the transcription level of any gene harboring an R6 motif.

Here, we have provided data to show that the R6 sequence is an adaptable motif for the elevation of transcription. This elevation will be concomitant to the expression of a MYB10-like gene, which can provide a temporal-spatial dimension since MYB10 is likely to be elevated itself by environmental and developmental cues, such as the onset of fruit ripening in specific skin cells. Enhanced color in apple and pear could be achieved via cis-genesis, or gene editing. This could enhance potential health components, as evidence suggests that a highanthocyanin diet may be beneficial (Espley et al., 2014), and that some anthocyanins are more bioactive in the diet than others (Nyanhanda et al., 2014). Other phytochemicals related to health, flavor or other quality parameters could be increased concurrent with color change, such as vitamin $\mathrm{C}$ concentration. In summary, the R6 motif is a novel and versatile tool for biotechnological approaches for plant manipulation.

\section{EXPERIMENTAL PROCEDURES}

\section{Plant Material and Transformation}

Arabidopsis plants (Columbia-0) were transformed using the floral dip method (Clough and Bent, 1998). Resultant transgenic seeds were sterilized in $70 \%$ ethanol $-0.05 \%$ Triton X-100 for $10 \mathrm{~min}$, then in $100 \%$ ethanol twice for $5 \mathrm{~min}$ each time. Seeds were dried on Whatman filter paper, then sprinkled onto growth media containing $1 / 2 \mathrm{MS}+2 \%$ sucrose $+50 \mathrm{mg} / \mathrm{L}$ kanamycin and placed at $25^{\circ} \mathrm{C}$ with $16 \mathrm{~h}$ photoperiod. At least three independent T2 generation lines were selected for analysis. Four-week-old transgenic seedlings were harvested and frozen in liquid nitrogen, ground into powder and stored at $-80^{\circ} \mathrm{C}$ for HPLC and $\mathrm{qPCR}$ analysis.

Nicotiana benthamiana and $N$. tabacum were grown in a glasshouse at $22-25^{\circ} \mathrm{C}$ with natural light and supplemental lighting to extend the day length to $16 \mathrm{~h}$.

Pear plants, cv. 'Conference,' were grown in vitro in apple multiplication media. Freshly opened leaves from 4 weeks old plants in tissue culture were used as explants for Agrobacteriummediated transformation based on the apple protocols (Yao et al., 2013). The vitamins in the apple protocols were replaced with the vitamin as described by Brisset et al. (1988). Transgenic plants were rooted and grown in a containment glasshouse at The New Zealand Institute for Plant and Food Research Limited (PFR), Auckland, New Zealand.

\section{Plasmid Construction}

Apple MYB10-R1 $1_{\text {pro }}$ and MYB10-R6 $6_{\text {pro }}$ promoters cloned in the pGreen0800LUC vector (Hellens et al., 2005) as a transcriptional fusion to the luciferase gene were previously described (Espley et al., 2009). Other constructs expressing the apple TFs and other MYB10 orthologs under the control of the CaMV35S promoter have also been described previously: MdMYB10, MdbHLH3, MdMYB8 (Espley et al., 2007), MdMYB110 (Chagne et al., 2013), PcMYB10, FaMYB10 and AtbHLH2/At1g63650/EGL3 (Lin-Wang et al., 2010), PhDPL (Albert et al., 2011), AtMYB75, MdMYB4, MdMYB6, MdMYB7, MdMYB11, MdMYB13, MdMYB14, MdMYB18, MdMYB19, MdMYB20, MdMYB21, MdMYB22, MdMYB24, MdMYB91, MdMYB92 (Hellens et al., 2005), MdMYB15, MdMYB16, MdMYB17, MdMYB111 (Lin-Wang et al., 2011), AcMYB110 (Montefiori et al., 2015). GenBank $^{\circledR}$ accessions for the TFs which were cloned into the pSAK277 vector under the control of the CaMV 35S promoter and not previously reported are as follows: Kiwifruit bHLH5 (KY623715), MdMYB3 (ADL36760.1), MdMYB5 (ADL36769.1), MdMYB9 (ABB84757.1), MdMYB12 (ADL36755.1), MdMYB28 (KY623714), MdMYB47 (ABK20308.1), MdMYB88 (ADL36771.1), MdMYB93 (ADL36772.1).

\section{Heterologous Promoter Engineering}

Promoters were engineered using standard restriction cloning of the PCR amplified R6 fragment from apple. A 1227 bp fragment of the AtMYB75 promoter was cloned into pGreen0800LUC in which the HindIII restriction site had been disrupted by digestion, T4 DNA polymerase treatment and re-ligation. The R6 motif was amplified from the R6:MYB10 genomic construct (Espley et al., 2009) using primers FW01 and RV01 (Supplementary Table S1), the PCR product was digested by DraI, the 194 bp fragment was gel purified, treated by T4 DNA polymerase and ligated into pGreen0800LUC-AtMYB75 $5_{\text {pro }}$ at the HindIII site located 489 bp upstream of the start codon to generate the AtMYB75-R6 $6_{\text {pro-LUC }}$ 
construct. The AtMYB75 promoters with and without the R6 insert were then excised from the pGreen0800-LUC (KpnI/NcoI) and used to replace the $35 \mathrm{~S}$ promoter in the pGreenII 62-SKAtMYB75 (35S-AtMYB75) to generate the R0-AtMYB75 and R6-AtMYB75 constructs.

A $3.3 \mathrm{~kb}$ fragment of the PcMYB10 promoter and the fulllength PcMYB10 gene, including $3.3 \mathrm{~kb}$ of promoter and $1.5 \mathrm{~kb}$ of terminator, were amplified from 'Williams Bon Chretien' pear genomic DNA using primers FW02/RV02 and FW02/RV03, and cloned into pGreen0800LUC and in pGEMTeasy, respectively. The 194 bp R6 fragment isolated as previously was ligated at the $B s g I$ restriction site located at position -275 within the PcMYB10 promoter in the pGreenLUC and in the pGEMTeasy constructs. The full-length PcMYB10 alleles with and without the R6 insert were then sub-cloned at the NotI site into the pART27 binary vector to generate the R1-PcMYB10 and R6-PcMYB10 constructs.

A full-length $F 3^{\prime} 5^{\prime} H$ gene, including $1906 \mathrm{bp}$ of promoter and $780 \mathrm{bp}$ of terminator was amplified from kiwifruit (A. chinensis) genomic DNA using primers FW03 and RV04 and cloned into pENTR-D/TOPO following instructions from the manufacturer (Invitrogen, Carlsbad, CA, United States). The plasmid was linearised at position -215 in the $F^{\prime} 5^{\prime} H$ promoter by inverse PCR using primers FW04 and RV05 and one, two and five copies of the R6 fragment, isolated as described earlier, was ligated within the promoter, hence generating new R6-, R12-, and R30- F $3^{\prime} 5^{\prime} H$ versions. The CaMV35S promoter region and nopaline synthase $3^{\prime}$ region were deleted from the pHEX2 vector (Hellens et al., 2005) to generate the pXCB2 vector in which the native (R0), R6, R12, and R30 alleles of $F 3^{\prime} 5^{\prime} H$ were recombined by LR reaction following manufacturer instructions (Invitrogen). The promoter region of the four $F 3^{\prime} 5^{\prime} H$ alleles was amplified using primers FW03 and RV06 and cloned at the BamHI$\mathrm{NcoI}$ sites fused to the LUC reporter in the pGreen0800LUC vector.

Cloning of the kiwifruit GGP promoter into pGreen0800LUC has been described previously (Laing et al., 2015). The R6 fragment isolated as described above was ligated at the $\mathrm{HpaI}$ restriction site located at position -694 within the GGP promoter. Positive clones were sequenced and a clone containing two tandem copies of the R6 fragment was selected (R12). The GGP promoter containing the R12 domain was fused to the GGP coding region by overlap PCR extension. The GGP promoter was amplified using primers OE3/OE4 and the GGP coding sequence was amplified from the pHEX2-GGP construct (Bulley et al., 2012) using OE1/OE2. Equal amount of the two gel-purified PCR products were mixed and used as template to amplify the full-length GGP allele using primers OE3/OE2, which was cloned into the pGreen0000 vector (Hellens et al., $2005)$ at the KpnI site. The non-canonical start codon of the uORF within the $5^{\prime}$ UTR of the GGP promoter was mutated from ACG to TTG by inverse PCR of the pGreen0000-R12:GGP construct using primers M1/M2 both containing the mutated TTG codon encompassed in an 18 bp overlap. Linearised mutated amplicon was re-ligated using In-Fusion HD enzyme (Clontech Laboratories, Inc., Mountain View, CA, United States) following instructions from the manufacturer.

\section{Phylogenetic Analysis}

The phylogenetic analysis was conducted using the PHYLIP suite of programs (Felsenstein, 1989). Full-length deduced amino acid sequences of 132 and 58 members of the MYB family from Arabidopsis and apple, respectively (sequences provided in Supplementary Date Sheet 1) were aligned using CLUSTALX (Thompson et al., 1997) and protein distances were calculated with PRODIST using the Jones-Taylor-Thornton matrix. Finally, the phylogenetic tree was generated by the neighbor-joining method and visualized using MEGA 4.0 software (Tamura et al., 2007).

\section{Transient Expression Assays}

The dual luciferase promoter assays in $N$. benthamiana and transient expression experiments in $N$. tabacum of the $\mathrm{F}^{\prime} 5^{\prime} \mathrm{H}$ and MYB constructs were performed as previously described (Espley et al., 2007). Tobacco infiltrated leaf areas were harvested 5 days after infiltration and cyanidin and delphinidin derivatives were analyzed by LC-MS. Transient expression in A. eriantha fruits was performed as described previously (Montefiori et al., 2011). Four mature fruits were syringe-injected with Agrobacterium suspensions containing the combination of constructs as indicated in the tables and figures. Tissue from the area of injection showing anthocyanin accumulation was collected at 6 days postinjection and anthocyanin was extracted and analyzed by LCMS. For ascorbate concentration experiments, $N$. tabacum leaves were co-infiltrated with equal amounts of Agrobacterium suspensions containing the GGP and the MYB constructs together with a $35 S_{p r o}: G M E$ (GDP-mannose-3',5'-epimerase) construct shown previously to synergistically enhance the ascorbate production by GGP (Bulley et al., 2009) and the suppressor of silencing P19, following a procedure previously described (Laing et al., 2015). Ascorbate content in leaf tissue was measured by HPLC as described in Rassam and Laing (2005).

\section{HPLC and LC-MS Analysis}

For each Arabidopsis line, three seedlings were snap-frozen in liquid nitrogen, powdered and freeze-dried overnight in the dark. The freeze-dried samples were then re-suspended in 100\% methanol $+0.1 \% \mathrm{HCl}$ and incubated at room temperature for $2 \mathrm{~h}$ in the dark with occasional vortexing. The samples were centrifuged and supernatants were spin dried for $2 \mathrm{~h}$, resuspended thoroughly in $20 \%$ methanol, centrifuged again and the supernatants were analyzed by LC-MS (Supplementary Data Sheet 2).

For each pear line, three mature fully expanded leaves were snap-frozen, powdered, and freeze-dried overnight. Samples were then extracted overnight at $1^{\circ} \mathrm{C}$ with $2.5 \mathrm{~mL}$ solvent (ethanol/water/formic acid 80/20/1 v/v/v). After the samples were centrifuged (Jouan, microcentrifuge and diluted with methanol/water/formic acid 50/50/5 v/v/v) the samples were analyzed by UHPLC as detailed in Supplementary Data Sheet 2 .

Nicotiana tabacum leaf samples and A. eriantha fruit samples expressing $\mathrm{F}^{\prime} 5^{\prime} \mathrm{H}$ constructs were harvested, snap-frozen in 
liquid nitrogen and freeze-dried overnight, then re-suspended in methanol $+0.1 \% \mathrm{HCl}$ and incubated at room temperature for $2 \mathrm{~h}$ and analyzed by UHPLC (Supplementary Data Sheet 2).

\section{Real-time Quantitative PCR (q-PCR) Analysis}

RNA from the 27 transgenic Arabidopsis samples and the 15 pear samples (fully expanded mature leaves) used for anthocyanin extractions were extracted by using Spectrum Plant Total RNA Kit (Sigma-Aldrich, St. Louis, MO, United States). First-strand cDNA synthesis was carried out by using RT Primer Mix according to manufacturer's instructions (QuantiTect Reverse Transcription kit, Qiagen, Hilden, Germany). Real-time qPCR DNA amplification and analysis was carried out using the LightCycler 480 Real-Time PCR System (Roche Diagnostics, Mannheim, Germany), with LightCycler 480 software version 1.5. The LightCycler 480 SYBR Green I Master Mix (Roche) was used, and $10 \mu \mathrm{l}$ of total reaction volume applied in all the reactions following the manufacturer's method. qPCR conditions were $5 \mathrm{~min}$ at $95^{\circ} \mathrm{C}$, followed by 40 cycles of $5 \mathrm{~s}$ at $95^{\circ} \mathrm{C}, 5 \mathrm{~s}$ at $60^{\circ} \mathrm{C}$, and $10 \mathrm{~s}$ at $72^{\circ} \mathrm{C}$, followed by $65-95^{\circ} \mathrm{C}$ melting curve detection. The qPCR efficiency of each gene was obtained by analysing the standard curve of a cDNA serial dilution of that gene. Sequence of the primers used are provided in Supplementary Table S1.

\section{AUTHOR CONTRIBUTIONS}

$\mathrm{AA}, \mathrm{RE}, \mathrm{CB}$, and $\mathrm{RH}$ conceived the project and its components. $\mathrm{CB}, \mathrm{KL}-\mathrm{W}, \mathrm{WL}$, and $\mathrm{SD}$ cloned constructs and performed transient transformations. ST generated pear lines. $\mathrm{CB}$ and KL-W conducted gene expression analysis. TM, WL, and YP grew Arabidopsis lines and performed HPLC. AA and $\mathrm{RE}$ performed bioinformatics. $\mathrm{CB}, \mathrm{AA}$, and $\mathrm{RE}$ wrote the paper.

\section{REFERENCES}

Albert, N. W., Davies, K. M., Lewis, D. H., Zhang, H., Montefiori, M., Brendolise, C., et al. (2014). A conserved network of transcriptional activators and repressors regulates anthocyanin pigmentation in eudicots. Plant Cell 26, 962-980. doi: 10.1105/tpc.113.122069

Albert, N. W., Lewis, D. H., Zhang, H., Schwinn, K. E., Jameson, P. E., and Davies, K. M. (2011). Members of an R2R3-MYB transcription factor family in Petunia are developmentally and environmentally regulated to control complex floral and vegetative pigmentation patterning. Plant J. 65, 771-784. doi: 10.1111/j. 1365-313X.2010.04465.x

Baldoni, E., Genga, A., Medici, A., Coraggio, I., and Locatelli, F. (2013). The OsMyb4 gene family: stress response and transcriptional auto-regulation mechanisms. Biol. Plant. 57, 691-700. doi: 10.1007/s10535-013-0331-3

Ban, Y., Honda, C., Hatsuyama, Y., Igarashi, M., Bessho, H., and Moriguchi, T. (2007). Isolation and functional analysis of a MYB transcription factor gene that is a key regulator for the development of red coloration in apple skin. Plant Cell Physiol. 48, 958-970. doi: 10.1093/pcp/pcm066

Baudry, A., Caboche, M., and Lepiniec, L. (2006). TT8 controls its own expression in a feedback regulation involving TTG1 and homologous MYB and bHLH factors, allowing a strong and cell-specific accumulation of flavonoids in

\section{ACKNOWLEDGMENTS}

We thank G. Wadasinghe for all his efforts in establishing pear grafts of the transgenic lines and Monica Dragulescu for expert maintenance of plants in the glasshouse. We also thank Nick Albert for his PhDPL construct and Mirco Montefiori for the AcMYB110 and AcbHLH5 constructs. Finally, we thank Nick Albert for reviewing the manuscript.

\section{SUPPLEMENTARY MATERIAL}

The Supplementary Material for this article can be found online at: https://www.frontiersin.org/articles/10.3389/fpls.2017.01864/ full\#supplementary-material

FIGURE S1 | Effect of the insertion of the R6 motif into the AcF3' $5^{\prime} \mathrm{H}$ promoter. (A) Schematic of the engineered $\mathrm{F}^{\prime} 5^{\prime} \mathrm{H}$ promoter fused to the $L U C$ reporter gene in pGreen0800LUC. (B) Dual luciferase promoter assay in N. benthamiana. Promoters, with or without the R6 motif, were co-infiltrated with MdMYB10 and MdbHLH3, or AcMYB110 alone. Luminescence of LUC and REN was measured 3 days post-infiltration and expressed as a ratio of LUC to REN. Data represent means ( \pm SE) of four technical replicate reactions. (C) Transient expression in $N$. tabacum of the $\mathrm{F}^{\prime} 5^{\prime} \mathrm{H}$ gene placed under the control of its own promoter $\left(F 3^{\prime} 5^{\prime} H_{\text {pro }}: F 3^{\prime} 5^{\prime} H\right)$, the R6-engineered $\mathrm{F}^{\prime} 5^{\prime} \mathrm{H}$ promoter $\left(F 3^{\prime} 5^{\prime} H-R 6_{p r o}: F 3^{\prime} 5^{\prime} H\right)$ or the 35S promoter (35S pro:F3' $5^{\prime} H$ ), in presence of AcMYB110. Cyanidin and delphinidin derivatives extracted from tobacco leaves are expressed as concentration of Cy-glu equivalent and (D) presented as a ratio of delphinidin to cyanidin.

FIGURE S2 | The number of R1 motifs affect the level of transactivation by MYB110. (A) $F 3^{\prime} 5^{\prime} H$ promoter, containing 0, 6, 12, or 30 repeats of the R1 motif, fused to the LUC reporter was co-infiltrated with MYB110 in N. benthamiana, and the LUC/REN ratio was calculated as previously. (B) Cyanidin and delphinidin derivatives extracted from tobacco leaves expressing the corresponding engineered promoters driving the $\mathrm{F}^{\prime} 5^{\prime} \mathrm{H}$ gene were quantified by HPLC and are presented as a ratio of delphinidin to cyanidin (Dp/Cy).

FIGURE S3 | ClustalW alignment of the R1 motif from Rosaceous species, Arabidopsis and Actinidia (A). Box represents required nucleotides for MYB10 binding (Espley et al., 2009). Graphical representation of the conserved residues at each position of the R1 motif was created in WebLogo (Crooks et al., 2004) (B), the height of each letter representing the relative frequency at that position.

Arabidopsis thaliana. Plant J. 46, 768-779. doi: 10.1111/j.1365-313X.2006. 02733.x

Boase, M., Brendolise, C., Wang, L., Ngo, H., Espley, R., Hellens, R., et al. (2015). Failure to launch: the self-regulating Md-MYB10 R6 gene from apple is active in flowers but not leaves of Petunia. Plant Cell Rep. 34, 1817-1823. doi: 10.1007/ s00299-015-1827-4

Borevitz, J. O., Xia, Y., Blount, J., Dixon, R. A., and Lamb, C. (2000). Activation tagging identifies a conserved MYB regulator of phenylpropanoid biosynthesis. Plant Cell 12, 2383-2394. doi: 10.1105/tpc.12.12.2383

Brisset, M.-N., Paulin, J.-P., and Duron, M. (1988). Feasibility of rating fire blight susceptibility of pear cultivars (Pyrus communis) on in vitro microcuttings. Agronomie 8, 707-710. doi: 10.1051/agro:19880806

Broun, P. (2004). Transcription factors as tools for metabolic engineering in plants. Curr. Opin. Plant Biol. 7, 202-209. doi: 10.1016/j.pbi.2004.01.013

Bulley, S., and Laing, W. (2016). The regulation of ascorbate biosynthesis. Curr. Opin. Plant Biol. 33, 15-22. doi: 10.1016/j.pbi.2016.04.010

Bulley, S., Wright, M., Rommens, C., Yan, H., Rassam, M., Lin-Wang, K., et al. (2012). Enhancing ascorbate in fruits and tubers through over-expression of the L-galactose pathway gene GDP-L-galactose phosphorylase. Plant Biotechnol. J. 10, 390-397. doi: 10.1111/j.1467-7652.2011. 00668.x 
Bulley, S. M., Rassam, M., Hoser, D., Otto, W., Schunemann, N., Wright, M., et al. (2009). Gene expression studies in kiwifruit and gene over-expression in Arabidopsis indicates that GDP-L-galactose guanyltransferase is a major control point of vitamin C biosynthesis. J. Exp. Bot. 60, 765-778. doi: 10.1093/jxb/ ern 327

Cao, J., Schneeberger, K., Ossowski, S., Gunther, T., Bender, S., Fitz, J., et al. (2011). Whole-genome sequencing of multiple Arabidopsis thaliana populations. Nat. Genet. 43, 956-963. doi: 10.1038/ng.911

Chagne, D., Lin-Wang, K., Espley, R. V., Volz, R. K., How, N. M., Rouse, S., et al. (2013). An ancient duplication of apple MYB transcription factors is responsible for novel red fruit-flesh phenotypes. Plant Physiol. 161, 225-239. doi: 10.1104/pp.112.206771

Chiu, L. W., Zhou, X., Burke, S., Wu, X., Prior, R. L., and Li, L. (2010). The purple cauliflower arises from activation of a MYB transcription factor. Plant Physiol. 154, 1470-1480. doi: 10.1104/pp.110.164160

Clough, S. J., and Bent, A. F. (1998). Floral dip: a simplified method for Agrobacterium-mediated transformation of Arabidopsis thaliana. Plant J. 16, 735-743. doi: 10.1046/j.1365-313x.1998.00343.x

Crooks, G. E., Hon, G., Chandonia, J. M., and Brenner, S. E. (2004). WebLogo: a sequence logo generator. Genome Res. 14, 1188-1190. doi: 10.1101/gr.849004

Daccord, N., Celton, J.-M., Linsmith, G., Becker, C., Choisne, N., Schijlen, E., et al. (2017). High-quality de novo assembly of the apple genome and methylome dynamics of early fruit development. Nat. Genet. 49, 1099-1106. doi: 10.1038/ ng.3886

Dare, A. P., Schaffer, R. J., Lin-Wang, K., Allan, A. C., and Hellens, R. P. (2008) Identification of a cis-regulatory element by transient analysis of co-ordinately regulated genes. Plant Methods 4:17. doi: 10.1186/1746-4811-4-17

Davuluri, R. V., Sun, H., Palaniswamy, S. K., Matthews, N., Molina, C., Kurtz, M., et al. (2003). AGRIS: Arabidopsis gene regulatory information server, an information resource of Arabidopsis cis-regulatory elements and transcription factors. BMC Bioinformatics 4:25. doi: 10.1186/1471-2105-4-25

Doudna, J. A., and Charpentier, E. (2014). The new frontier of genome engineering with CRISPR-Cas9. Science 346:1258096. doi: 10.1126/science.1258096

Du, H., Yang, S.-S., Liang, Z., Feng, B.-R., Liu, L., Huang, Y.-B., et al. (2012). Genome-wide analysis of the MYB transcription factor superfamily in soybean. BMC Plant Biol. 12:106. doi: 10.1186/1471-2229-12-106

Dubos, C., Le Gourrierec, J., Baudry, A., Huep, G., Lanet, E., Debeaujon, I., et al. (2008). MYBL2 is a new regulator of flavonoid biosynthesis in Arabidopsis thaliana. Plant J. 55, 940-953. doi: 10.1111/j.1365-313X.2008.03564.x

Dubos, C., Stracke, R., Grotewold, E., Weisshaar, B., Martin, C., and Lepiniec, L. (2010). MYB transcription factors in Arabidopsis. Trends Plant Sci. 15, 573-581. doi: 10.1016/j.tplants.2010.06.005

Espley, R. V., Brendolise, C., Chagne, D., Kutty-Amma, S., Green, S., Volz, R., et al. (2009). Multiple repeats of a promoter segment causes transcription factor autoregulation in red apples. Plant Cell 21, 168-183. doi: 10.1105/tpc 108.059329

Espley, R. V., Butts, C. A., Laing, W. A., Martell, S., Smith, H., McGhie, T. K., et al. (2014). Dietary flavonoids from modified apple reduce inflammation markers and modulate gut microbiota in mice. J. Nutr. 144, 146-154. doi: 10.3945/jn. 113.182659

Espley, R. V., Hellens, R. P., Putterill, J., Stevenson, D. E., Kutty-Amma, S., and Allan, A. C. (2007). Red colouration in apple fruit is due to the activity of the MYB transcription factor, MdMYB10. Plant J. 49, 414-427. doi: 10.1111/j.1365313X.2006.02964.X

Felsenstein, J. (1989). PHYLIP - phylogeny inference package (Version 3.2). Cladistics 5, 164-166.

Fraser, L. G., Seal, A. G., Montefiori, M., McGhie, T. K., Tsang, G. K., Datson, P. M., et al. (2013). An R2R3 MYB transcription factor determines red petal colour in an Actinidia (kiwifruit) hybrid population. BMC Genomics 14:28. doi: 10.1186/1471-2164-14-28

Fukui, Y., Tanaka, Y., Kusumi, T., Iwashita, T., and Nomoto, K. (2003). A rationale for the shift in colour towards blue in transgenic carnation flowers expressing the flavonoid $3^{\prime}, 5^{\prime}$-hydroxylase gene. Phytochemistry 63, 15-23. doi: 10.1016/ S0031-9422(02)00684-2

Grotewold, E., Sainz, M. B., Tagliani, L., Hernandez, J. M., Bowen, B., and Chandler, V. L. (2000). Identification of the residues in the Myb domain of maize C1 that specify the interaction with the bHLH cofactor R. Proc. Natl. Acad. Sci. U.S.A. 97, 13579-13584. doi: 10.1073/pnas.250379897
Gu, C., Liao, L., Zhou, H., Wang, L., Deng, X., and Han, Y. (2015). Constitutive activation of an anthocyanin regulatory gene PcMYB10.6 is related to red coloration in purple-foliage plum. PLOS ONE 10:e0135159. doi: 10.1371/ journal.pone.0135159

Harborne, J. (1967). Comparative Biochemistry of the Flavonoids. London: Academic Press.

Hartmann, U., Sagasser, M., Mehrtens, F., Stracke, R., and Weisshaar, B. (2005). Differential combinatorial interactions of cis-acting elements recognized by R2R3-MYB, BZIP, and BHLH factors control light-responsive and tissuespecific activation of phenylpropanoid biosynthesis genes. Plant Mol. Biol. 57, 155-171. doi: 10.1007/s11103-004-6910-0

Hellens, R. P., Allan, A. C., Friel, E. N., Bolitho, K., Grafton, K., Templeton, M. D., et al. (2005). Transient expression vectors for functional genomics, quantification of promoter activity and RNA silencing in plants. Plant Methods 1:13. doi: 10.1186/1746-4811-1-13

Heppel, S. C., Jaffe, F. W., Takos, A. M., Schellmann, S., Rausch, T., Walker, A. R., et al. (2013). Identification of key amino acids for the evolution of promoter target specificity of anthocyanin and proanthocyanidin regulating MYB factors. Plant Mol. Biol. 82, 457-471. doi: 10.1007/s11103-013-0074-8

Higo, K., Ugawa, Y., Iwamoto, M., and Korenaga, T. (1999). Plant cis-acting regulatory DNA elements (PLACE) database: 1999. Nucleic Acids Res. 27, 297-300. doi: 10.1093/nar/27.1.297

Hobert, O. (2008). Gene regulation by transcription factors and microRNAs. Science 319, 1785-1786. doi: 10.1126/science.1151651

Holme, I. B., Wendt, T., and Holm, P. B. (2013). Intragenesis and cisgenesis as alternatives to transgenic crop development. Plant Biotechnol. J. 11, 395-407. doi: $10.1111 /$ pbi. 12055

Jin, H., and Martin, C. (1999). Multifunctionality and diversity within the plant MYB-gene family. Plant Mol. Biol. 41, 577-585. doi: 10.1023/A:1006319732410 Katsumoto, Y., Fukuchi-Mizutani, M., Fukui, Y., Brugliera, F., Holton, T. A., Karan, M., et al. (2007). Engineering of the rose flavonoid biosynthetic pathway successfully generated blue-hued flowers accumulating delphinidin. Plant Cell Physiol. 48, 1589-1600. doi: 10.1093/pcp/pcm131

Korkuc, P., Schippers, J. H. M., and Walther, D. (2014). Characterization and identification of cis-regulatory elements in arabidopsis based on single-nucleotide polymorphism information. Plant Physiol. 164, 181-200. doi: 10.1104/pp.113.229716

Lai, Y., Li, H., and Yamagishi, M. (2013). A review of target gene specificity of flavonoid R2R3-MYB transcription factors and a discussion of factors contributing to the target gene selectivity. Front. Biol. 8, 577-598. doi: 10.1007/ s11515-013-1281-z

Laing, W. A., Martinez-Sanchez, M., Wright, M. A., Bulley, S. M., Brewster, D., Dare, A. P., et al. (2015). An upstream open reading frame is essential for feedback regulation of ascorbate biosynthesis in Arabidopsis. Plant Cell 27, 772-786. doi: 10.1105/tpc.114.133777

Li, S. F., Milliken, O. N., Pham, H., Seyit, R., Napoli, R., Preston, J., et al. (2009). The Arabidopsis MYB5 transcription factor regulates mucilage synthesis, seed coat development, and trichome morphogenesis. Plant Cell 21, 72-89. doi: 10.1105/tpc.108.063503

Lin-Wang, K., Bolitho, K., Grafton, K., Kortstee, A., Karunairetnam, S., McGhie, T. K., et al. (2010). An R2R3 MYB transcription factor associated with regulation of the anthocyanin biosynthetic pathway in Rosaceae. BMC Plant Biol. 10:50. doi: 10.1186/1471-2229-10-50

Lin-Wang, K., Micheletti, D., Palmer, J., Volz, R., Lozano, L., Espley, R., et al. (2011). High temperature reduces apple fruit colour via modulation of the anthocyanin regulatory complex. Plant Cell Environ. 34, 1176-1190. doi: 10.1111/j.1365-3040.2011.02316.x

Menkens, A. E., Schindler, U., and Cashmore, A. R. (1995). The G-box: a ubiquitous regulatory DNA element in plants bound by the GBF family of bZIP proteins. Trends Biochem. Sci. 20, 506-510. doi: 10.1016/S0968-0004(00) 89118-5

Montefiori, M., Brendolise, C., Dare, A. P., Lin-Wang, K., Davies, K. M., Hellens, R. P., et al. (2015). In the Solanaceae, a hierarchy of bHLHs confer distinct target specificity to the anthocyanin regulatory complex. J. Exp. Bot. 66, 1427-1436. doi: $10.1093 /$ jxb/eru494

Montefiori, M., Comeskey, D. J., Wohlers, M., and McGhie, T. K. (2009). Characterization and quantification of anthocyanins in red kiwifruit (Actinidia spp.). J. Agric. Food Chem. 57, 6856-6861. doi: 10.1021/jf900800z 
Montefiori, M., Espley, R. V., Stevenson, D., Cooney, J., Datson, P. M., Saiz, A., et al. (2011). Identification and characterisation of F3GT1 and F3GGT1, two glycosyltransferases responsible for anthocyanin biosynthesis in red-fleshed kiwifruit (Actinidia chinensis). Plant J. 65, 106-118. doi: 10.1111/j.1365-313X. 2010.04409.x

Nyanhanda, T., Gould, E. M., McGhie, T., Shaw, O. M., Harper, J. L., and Hurst, R. D. (2014). Blackcurrant cultivar polyphenolic extracts suppress CCL26 secretion from alveolar epithelial cells. Food Funct. 5, 671-677. doi: 10.1039/ c3fo60568j

Okinaka, Y., Shimada, Y., Nakano-Shimada, R., Ohbayashi, M., Kiyokawa, S., and Kikuchi, Y. (2003). Selective accumulation of delphinidin derivatives in tobacco using a putative flavonoid $3^{\prime}, 5^{\prime}$-hydroxylase cDNA from Campanula medium. Biosci. Biotechnol. Biochem. 67, 161-165. doi: 10.1271/bbb.67.161

Peng, T., Saito, T., Honda, C., Ban, Y., Kondo, S., Liu, J. H., et al. (2013). Screening of UV-B-induced genes from apple peels by SSH: possible involvement of MdCOP1-mediated signaling cascade genes in anthocyanin accumulation. Physiol. Plant. 148, 432-444. doi: 10.1111/ppl.12002

Ptashne, M. (1988). How eukaryotic transcriptional activators work. Nature 335, 683-689. doi: 10.1038/335683a0

Ramsay, N. A., and Glover, B. J. (2005). MYB-bHLH-WD40 protein complex and the evolution of cellular diversity. Trends Plant Sci. 10, 63-70. doi: 10.1016/j. tplants.2004.12.011

Rassam, M., and Laing, W. (2005). Variation in ascorbic acid and oxalate levels in the fruit of Actinidia chinensis tissues and genotypes. J. Agric. Food Chem. 53, 2322-2326. doi: 10.1021/jf048197s

Ravaglia, D., Espley, R. V., Henry-Kirk, R. A., Andreotti, C., Ziosi, V., Hellens, R. P., et al. (2013). Transcriptional regulation of flavonoid biosynthesis in nectarine (Prunus persica) by a set of R2R3 MYB transcription factors. BMC Plant Biol. 13:68. doi: 10.1186/1471-2229-13-68

Riechmann, J. L. (2002). Transcriptional regulation: a genomic overview. Arabidopsis Book 1:e0085. doi: 10.1199/tab.0085

Riechmann, J. L., Krizek, B. A., and Meyerowitz, E. M. (1996). Dimerization specificity of Arabidopsis MADS domain homeotic proteins APETALA1, APETAlA3, PISTILlATA, and AGAMOUS. Proc. Natl. Acad. Sci. U.S.A. 93, 4793-4798. doi: 10.1073/pnas.93.10.4793

Rombauts, S., Florquin, K., Lescot, M., Marchal, K., Rouze, P., and van de Peer, Y. (2003). Computational approaches to identify promoters and cis-regulatory elements in plant genomes. Plant Physiol. 132, 1162-1176. doi: 10.1104/pp.102. 017715

Romero, I., Fuertes, A., Benito, M. J., Malpica, J. M., Leyva, A., and Paz-Ares, J. (1998). More than 80R2R3-MYB regulatory genes in the genome of Arabidopsis thaliana. Plant J. 14, 273-284. doi: 10.1046/j.1365-313X.1998.00113.x

Sablowski, R. W. M., Moyano, E., Culianez-Macia, F. A., Schuch, W., Martin, C., and Bevan, M. (1994). A flower-specific Myb protein activates transcription of phenylpropanoid biosynthetic genes. EMBO J. 13, 128-137.

Seitz, C., Ameres, S., and Forkmann, G. (2007). Identification of the molecular basis for the functional difference between flavonoid $3^{\prime}$-hydroxylase and flavonoid 3',5'-hydroxylase. FEBS Lett. 581, 3429-3434. doi: 10.1016/j.febslet.2007.06.045

Stracke, R., Werber, M., and Weisshaar, B. (2001). The R2R3-MYB gene family in Arabidopsis thaliana. Curr. Opin. Plant Biol. 4, 447-456. doi: 10.1016/S13695266(00)00199-0

Takos, A. M., Jaffe, F. W., Jacob, S. R., Bogs, J., Robinson, S. P., and Walker, A. R. (2006). Light-induced expression of a MYB gene regulates anthocyanin biosynthesis in red apples. Plant Physiol. 142, 1216-1232. doi: 10.1104/pp.106. 088104

Tamura, K., Dudley, J., Nei, M., and Kumar, S. (2007). MEGA4: molecular evolutionary genetics analysis (MEGA) software version 4.0. Mol. Biol. Evol. 24, 1596-1599. doi: 10.1093/molbev/msm092
Thompson, J. D., Gibson, T. J., Plewniak, F., Jeanmougin, F., and Higgins, D. G. (1997). The ClustalX windows interface: flexible strategies for multiple sequence alignment aided by quality analysis tools. Nucleic Acids Res. 24, 4876-4882. doi: 10.1093/nar/25.24.4876

Tian, J., Peng, Z., Zhang, J., Song, T., Wan, H., Zhang, M., et al. (2015). McMYB10 regulates coloration via activating $\mathrm{McF}^{\prime} \mathrm{H}$ and later structural genes in ever-red leaf crabapple. Plant Biotechnol. J. 13, 948-961. doi: 10.1111/pbi. 12331

van Nocker, S., Berry, G., Najdowski, J., Michelutti, R., Luffman, M., Forsline, P. et al. (2011). Genetic diversity of red-fleshed apples (Malus). Euphytica 185, 281-293. doi: 10.1016/j.gene.2014.04.045

Velasco, R., Zharkikh, A., Affourtit, J., Dhingra, A., Cestaro, A., Kalyanaraman, A., et al. (2010). The genome of the domesticated apple (Malus $\times$ domestica Borkh.). Nat. Genet. 42, 833-839. doi: 10.1038/ng.654

Venter, M. (2007). Synthetic promoters: genetic control through cis engineering. Trends Plant Sci. 12, 118-124. doi: 10.1016/j.tplants.2007.01.002

Volz, R. K., Oraguzie, N. C., Whitworth, C. J., How, N., Chagné, D., Carlisle, C. M., et al. (2009). "Breeding for red flesh colour in apple: progress and challenges", in Proceedings of the International Society for Horticultural Science (ISHS), Leuven, 337-342. doi: 10.17660/ActaHortic.2009.814.54

Wilkins, O., Nahal, H., Foong, J., Provart, N. J., and Campbell, M. M. (2009). Expansion and diversification of the Populus R2R3-MYB family of transcription factors. Plant Physiol. 149, 981-993. doi: 10.1104/pp.108.132795

Wingender, E., Dietze, P., Karas, H., and Knuppel, R. (1996). TRANSFAC: a database on transcription factors and their DNA binding sites. Nucleic Acids Res. 24, 238-241. doi: 10.1093/nar/24.1.238

Xu, W., Grain, D., Le Gourrierec, J., Harscoët, E., Berger, A., Jauvion, V., et al. (2013). Regulation of flavonoid biosynthesis involves an unexpected complex transcriptional regulation of TT8 expression, in Arabidopsis. New Phytol. 198, 59-70. doi: 10.1111/nph.12142

Yao, J.-L., Tomes, S., and Gleave, A. P. (2013). Transformation of apple (Malus $\times$ domestica) using mutants of apple acetolactate synthase as a selectable marker and analysis of the T-DNA integration sites. Plant Cell Rep. 32, 703-714. doi: 10.1007/s00299-013-1404-7

Zhao, J., Zhang, W., Zhao, Y., Gong, X., Guo, L., Zhu, G., et al. (2007). SAD2, an importin $\beta$-like protein, is required for UV-B response in Arabidopsis by mediating MYB4 nuclear trafficking. Plant Cell 19, 3805-3818. doi: 10.1105/ tpc. 106.048900

Zhou, Y., Zhou, H., Lin-Wang, K., Vimolmangkang, S., Espley, R. V., Wang, L., et al. (2014). Transcriptome analysis and transient transformation suggest an ancient duplicated MYB transcription factor as a candidate gene for leaf red coloration in peach. BMC Plant Biol. 14:388. doi: 10.1186/s12870-0140388-y

Zhu, C., and Perry, S. E. (2005). Control of expression and autoregulation of AGL15, a member of the MADS-box family. Plant J. 41, 583-594. doi: 10.1111/ j.1365-313X.2004.02320.x

Conflict of Interest Statement: The authors declare that the research was conducted in the absence of any commercial or financial relationships that could be construed as a potential conflict of interest.

Copyright (C) 2017 Brendolise, Espley, Lin-Wang, Laing, Peng, McGhie, Dejnoprat, Tomes, Hellens and Allan. This is an open-access article distributed under the terms of the Creative Commons Attribution License (CC BY). The use, distribution or reproduction in other forums is permitted, provided the original author (s) or licensor are credited and that the original publication in this journal is cited, in accordance with accepted academic practice. No use, distribution or reproduction is permitted which does not comply with these terms. 